\title{
APPLICATIONS OF NANOMATERIALS IN THE MEAT AND SEAFOOD PROCESSING INDUSTRY
}

Montero, M.P.*, López-Caballero, M.E., Alemán, A., Gómez-Estaca, J. Martínez-Álvarez, Ó. and Gómez-Guillén M.C.

Instituto de Ciencia y Tecnología de los Alimentos y Nutrición (ICTAN). Consejo Superior de Investigaciones Científicas (CSIC)

C/ José Antonio Novais no 10, 28040 Madrid, Spain.

*Corresponding author: E-mail address: mpmontero@ictan.csic.es

ICTAN-CSIC maintains implemented a Quality Management System ISO standard 9001:2018.

\section{ABSTRACT}

The application of nanoparticles and nanostructures in meat and fishery products is relatively recent and is arousing increasing interest. Nanostructure magnifies the properties with respect to traditional compounds (which are larger), owing to the greater surface area exposed and the small size, facilitating greater accessibility in food. There are many different kinds of nanoparticles and nanostructures; for example, they may use metals, or substances of vegetable or animal origin, such as chitosan structures. Otherwise, the application of natural compounds to maintain product quality is increasingly demanded, but the sensory characteristics of the products limit their use. Encapsulation by nanovesicles (emulsions and liposomes) of these compounds is a good strategy to mitigate their sensory characteristics while allowing progressive release of the bioactive agent to exert its action. This is the case with essential oil nanoemulsions. Liposomes entrapping bioactive compounds of many different kinds could be ingredients for the design of functional food products, while they improve the nutritional value 
because to their high quantity polyunsaturated fatty acid, but they can also be used in the food industry for a large diversity of technological purposes. Meat and fishery product packaging can be improved by nanotechnology in aspects concerning the improvement of physicochemical properties and the design of active and intelligent packages.

With regard to the use of nanobiosensors, often they are mere prototypes, but no doubt they will soon be implemented in industrial quality control because they present high sensitivity, repeatability, and quickness. They are mainly applied to guarantee species identification, and to detect compounds and microorganisms.

Keywords: active nanoparticles, nanoliposomes, nanoemulsions, active nanocomposite packaging, nanobiosensors

\section{INTRODUCTION}

Consumer rejection of chemical additives and the increasing fondness for minimally processed foods that look like fresh and natural ones necessitate the use of technologies and/or techniques that ensure the quality and stability of food while at the same time guaranteeing their safety and security. The use of nanoscale elements is attracting more and more interest every day, used alone and/or as part of packages to improve their properties or endow them with activity (by interaction of nanocomposites with food or with the environment), as part of emulsion systems, nanocapsules, etc. (Singh et al 2016). The application of nanotechnology allows new applications, and improves the properties, being especially useful in the development of the products, among the possible applications is found it is worth highlighting: the modification of the flavors and texture, the improved absorption of nutrients, and better microorganism detection system, among other properties, as well as diversification in the food packaging system. 
Nanotechnology can be involved in various aspects related with both the development and the preservation of food products based on myosystems (meats, poultry and seafood). Basically, the following possibilities need to be considered: i) when adding an ingredient or additive in the nano size; in this sense, nano-sized metal structures are gaining increasing interest (MartínezAbad et al. (2012); ii) when the ingredient or additive is modified forming a nanostructure, -for example a phospholipid that forms a liposomal nanovesicle-, which could be used to encapsulate bioactive compounds (Marín et al 2018 a,b); iii) when any of the components of the protective cover (coating solutions or films) having nano size is used to confer special properties to the packaging. This would be the typical case of exfoliated clays as nano-reinforcements in films for biodegradable packaging (Echeverría et al.,2016), or the inclusion of nano-liposomes in edible films (Montero et al 2019). These are just a couple of examples, their applications in this field being extremely versatile. Finally, nanotechnology can also be applied for nanobiosensors construction (Inbaraj and Chen 2016, Duan et al. (2017).

The use of nanoparticles in the meat and seafood processing industry is the target of this chapter, which focuses mainly on improving the quality of myosystems (including formulation and use of by-products) and their preservation. Besides it also covers aspects related to the incorporation of nanoparticles in animal feed or even in nutraceuticals/drugs. Finally, it will also deal with of the use of biosensors, to the detection of compounds/analytes and microorganisms in a fast, effective manner to guarantee the quality of meat and fish products.

\section{ADDITION OF NANOESTRUCTURES IN MEAT AND FISH PRODUCTS}

\subsection{NON-ENCAPSULATING NANOPARTICLES}

Many works in the literature deal with the application of nanoparticles (NPs) not alone but incorporated in food packaging or containers, as will be discussed in Section 3. Despite this, as 
many of the articles base their discussion on the activity of the particles, it is considered interesting to mention them in this section, regardless of whether the particles are included in a physical support.

Among the various kinds of nanoparticles, metal nanomaterials are mainly used for their bactericidal properties. Silver $(\mathrm{Ag})$ nanoparticles are the ones that have been most studied and their use is subject to legislation in many countries, as mentioned below. Mahdi et al. (2012) found that in minced beef stored at $3 \pm 1{ }^{\circ} \mathrm{C}$ the shelf life ( 2 days) in usual food packaging was prolonged to 7 days, while the acceptability scores in nanosilver packaging (polyvinyl chloridepolyethylene laminate trays fortified by Ag NPs)were similar. These results were associated with the thickness of the meat, and the effect was striking in Staphylococcus aureus compared with Escherichia coli. As a result of the incorporation of 0.001-10 wt\% silver ions in an ethylene-vinyl alcohol (EVOH) copolymer matrix by a solvent casting technique, Martínez-Abad et al. (2012) obtained a high protein content food (chicken wings or breast and marinated pork loin) that showed low susceptibility to the films ( $<1$ log decrease) in terms of counts of Listeria monocytogenes when incubated 24 and $72 \mathrm{~h}$ at $12{ }^{\circ} \mathrm{C}$, In addition, vegetables (low protein content food displayed no noticeable bacterial counts for 1 and $10 \mathrm{wt} \%$ silver films and nearby 2 log reduction for $0.1 \mathrm{wt} \%$ silver films. In this work, the EVOH film ( $0.1 \%$ silver content) was not enough to guarantee that the products would not exceed the restriction limits recommended by EFSA (2011). Moreover, these authors stated that for Gram-positive and Gram-negative bacteria, the efficacy of antimicrobial silver is improved when incorporated in the copolymer $(\mathrm{EVOH})$ rather than being present as pure silver nitrate; this could be related to the inactivation of silver nanoparticles by the nutrient broth or by the microorganisms themselves. When in solution, all the silver ions could potentially be able of being immediately inactivated, while the release continues in the polymer for some time. This increases the interval of contact between the active silver ions and the bacteria, thus avoiding proliferation more efficiently. Rai et al. (2009) reported that Ag nanoparticles display effective antimicrobial properties bring into 
comparison to other salts owing to their particularly large surface area, which offers better connection with microorganisms. The nanoparticles become linked to the cell membrane and also enter inside the bacteria. The membrane of the bacteria includes sulfur-containing proteins; the Ag-nanoparticles engage with with these proteins and with phosphorus-containing compounds such as DNA. Once Ag-nanoparticles enter the bacterial cell, it is formed a low molecular weight region in the midpoint of the bacterium with which the bacterium conglomerates, thus preserving the DNA from the Ag-ions. The nanoparticles preferentially disrupt the respiratory chain, and cell division finally leads to death. Furthermore, Ag nanoparticles can be incorporated in target matrices in various ways (Toker, 2013), and they are stable and have low volatility (Youssef and Abdel-Aziz, 2013).

Fernández et al. (2010) developed by in situ reduction of silver nitrate (1\%) adsorbed on cellulose fibers by thermal and UV treatments silver-based antibacterial hybrid materials. These authors studied the ability of these materials to lower microbial contamination of exuded fluids during beef meat storage $\left(4 \pm 0.2^{\circ} \mathrm{C}, 10\right.$ days, with $12 \mathrm{~h}$ darkness and $12 \mathrm{~h}$ fluorescent light) in modified atmosphere packaging $\left(69.1 \% \mathrm{O}_{2}, 28.2 \% \mathrm{CO}_{2}\right.$, and $\left.2.7 \% \mathrm{~N}_{2}\right)$. Cellulose-silver hybrid materials decreased the total aerobic bacteria, lactic acid bacteria, pseudomonad spp., and enterobacteria found in the absorbent pads around $1 \mathrm{log} \mathrm{cfu} / \mathrm{g}$ during the storage. The total aerobic bacteria and Pseudomonas spp. In the presence of silver ions, counts for total aerobic bacteria and Pseudomonas spp. decreased considerably, while LAB were not significantly affected. Enterobacteriaceae continued under the detection threshold if silver was present. The meat color and the amount of microorganism were not noticeably changed by the silver-based materials. In beef meat exudates, the level of migrated silver ions reached $60 \mathrm{ppm}$. Regarding this, the EFSA (European Food Safety Authority) has recommended that the upper limit of Ag migration from packaging should not exceed $0.05 \mathrm{mg} / \mathrm{L}$ in water and $0.05 \mathrm{mg} / \mathrm{kg}$ in food. Therefore, assessments of silver migration would be necessary to ensure antimicrobial effectiveness in accordance with present legislation (Carbone et al., 2016). Meanwhile, the 
USFDA recommends, for all materials, that manufacturers should study and elaborate a toxicological profile for each container/packaging with nanomaterials (USFDA, 2014).

In addition to silver nanoparticles, other metals are also applied at the nano level to increase the quality of food. In turkey meat, Khalaf et al. (2013) evaluated the antimicrobial activities of pullulan (composed of maltotriose units) films incorporating $100 \mathrm{~nm}$ silver nanoparticles (Ag NPs) and $110 \mathrm{~nm}$ zinc oxide nanoparticles (ZnO NPs), applied on turkey deli meat that was previously inoculated with S. aureus and L. monocytogenes, and stored for two weeks $\left(4^{\circ} \mathrm{C}\right)$. The effect of essential oils (rosemary and oregano) was also tested. The Ag NP- and ZnO NP-based films gradually diminished the growing of both microorganisms from the first day of storage to values close to the limit of detection, with marked inhibition for S. aureus, and Ag NPs and/or edible films were more active than ZnO NPs and rosemary oil, respectively. These pullulan filmswere also tested on raw beef, turkey breast, and ready-to-eat turkey deli meat (previously inoculated with L. monocytogenes, S. typhimurium, S. aureus, and E. coli 0157:H7), under vacuum packaging during storage for 3 weeks at $4{ }^{\circ} \mathrm{C}$ (Morsy et al., 2014). Moreover, the application of these films on raw beef contaminated with S. typhimurium reduced the counts by as much as 4 logarithmic cycles or more. In another experiment, suspensions of zinc oxide ( $\mathrm{ZnO})$ (at 6 and $8 \mathrm{mM}$ ), containing 1\% acetic acid were examined against E. coli, S. aureus, B. cereus and L. monocytogenes, (Mirhosseini \& Arjmand, 2014). The results showed that suspensions of ZnO were effective against all the strains; furthermore, $\mathrm{ZnO}$ nanoparticles diminished initial growth when these species were inoculated in sheep meat. Silica nanoparticles (nano-SiO ${ }_{2}$, NSLDPE) (Luo et al., 2015a) and titanium nanoparticles (nano-TiO ${ }_{2}$, NTLDPE) (Luo et al., 2015b) were blended with low-density polyethylene (LDPE) to produce packaging (NSLDPE and NTLDPE, respectively) that was shown to be a promising alternative preservation process for shrimp preservation during chilled storage. These authors found that Pacific white shrimp packaged with NTLDPE or NSLDPE preserved freshness by reducing the total viable count, the total volatile base nitrogen (TVB-N) contents, thiobarbituric acid reactive substances (TBARS), and ATP 
degradation (K value) compared to the control (LDPE). The modified LDPE packaging also slowed the decrease of the water holding capacity that could might be related to the smaller degradation of muscle proteins) and the increase of the melanosis score by means of polyphenol oxidase inhibition.

Even though metal nanoparticles incorporated in packaging have been the main subject of study, especially in the last decade, plant-based nanoparticles such as pomegranate peel have also been studied in protein matrix. Lyophilized pomegranate peel nanoparticles (LPP NPs, $80 \pm$ $5 \mathrm{~nm}$ size) were incorporated at 1 and $1.5 \%$ in freshly minced beef meat that was stored for 15 days at $4 \pm 1{ }^{\circ} \mathrm{C}$ (Morsy et al., 2018). Butylated hydroxytoluene (BHT, $0.01 \%$ ) was used for control purposes. The LPP NPs exhibited a high phenolic content, and in the mentioned samples the contents of thiobarbituric acid reactive substances (TBARS), peroxides, and total volatile base nitrogen (TVB-N) were lower than in the control (up to 15 days), as well as the microbial load (total viable bacteria, psychrophilic, and lipolytic bacteria). Cooking characteristics of meatballs that included LPP NPs improved during conservation. Sensory evaluation of the parameters as rancid odor and color of those meatballs were acceptable and achieved a high score for up to 15 days. In another study, benzoic acid (phenolic compound) was encapsulated in polylactic-coglycolic acid nanoparticles (168.9 $\pm 0.5 \mathrm{~nm}$ ), which were injected into chicken meat (deboned fresh and cooked) (Ravichandran et al., 2011). The nanoparticles were effective against $S$. typhimurium (1.0 and $1.6 \mathrm{log} \mathrm{cfu} / \mathrm{g}$ decrease) and L. monocytogenes (1.1 and $3.2 \mathrm{log} \mathrm{cfu} / \mathrm{g}$ decrease) on 9 and 14 days of storage, respectively, compared with control samples (1.2 log cfu/g without nanoparticles). These authors stated that the lack of an outer cell wall and the accessible entrance of the nanoparticles and phenolics compounds into the bacteria could be the reasons for the higher reduction achieved in Listeria compared with Salmonella. Moreover, the greater inhibition in cooked compared with raw samples point to the absence of common microflora destroyed during cooking, which could have hindered the inhibitory characteristics of the nanoparticles. 
The antimicrobial effectiveness of citral as main compound of essential oil was improved by using nanostructured lipid carriers (NLCs) (Mokarizadeh et al., 2017). The citral interacted with the various ingredients (Mygliol, Precirol, and Poloramex), resulting in a high encapsulation efficiency, with particles that were stable for 90 days. These authors reported lower minimum inhibitory and bactericidal concentrations than those of citral emulsion for a selected group of microorganisms (S. aureus, B. cereus, E. coli, and Candida albicans); although they have not yet been applied in protein matrices, the encapsulation of essential oils in nanostructured lipid carriers could be a promising strategy to be applied in the food industry. The influence of garlic nanoparticles on the characteristics of beef burgers (premium and standard quality, raw and precooked, following the parameters established by Colombian Technical Standard (NTC) 1325 has also been studied (Torres-Vargas et al., 2016). These authors observed that the incorporation of garlic nanoparticles did not affect the structure of the precooked and raw burgers, but it produced an increase in the red tendency $\left(a^{*}\right)$ in the premium burgers. The cooking process showed a decrease in the $L$ * parameter of the formulations and an increment in shear force during storage. The microbiological analysis (mesophilic aerobic organisms, fecal and total coliforms, S. aureus, sulfite-reducing Clostridium spores and Salmonella sp.) fulfilled the parameters required by NTC 1325 .

In addition to preserving quality during storage, nanoscale materials have also been used to modify technological properties of fish proteins. Thus, Yin and Park (2014a) observed that the properties of gelation of Alaska pollock surimi after setting were influenced by the incorporation of nanoscale fish bone (NFB) at diverse concentrations $(0 \%, 0.1 \%, 0.25 \%, 0.5 \%, 1 \%$, and $2 \%)$. The nanoscale particles were too small to dissociate the myofibrillar protein matrix. These authors observed that the rheological parameters, as breaking distance and breaking force of these surimi gels after setting enlarged significantly as the concentration of nanoscale fish bone raised up to $1 \%$. Both light meromyosin chains and myosin heavy chains were aggregated to a larger extent as NFB concentration augmented. Thus, the first peak temperature and storage modulus 
value $\left(G^{\prime}\right)$, increased as NFN concentration increased (both related to the unfolding and aggregation of light meromyosin). Similarly, the intensity of myosin heavy chain cross-links also increased as NFB concentration increased. This fact could indicate the formation of additional $\varepsilon$-( $\gamma$-glutamyl) lysine covalent bond by means of endogenous transglutaminase (which also increased as NFB calcium concentration increased), and more calcium ions from NFB. The texture of the gel rose with up to $1 \%$ NFB, due to calcium released from NFB and endogenous transglutaminase. Moreover, these authors found that these results were affected by the particle size of the fish bone, as surimi with micro fish bone particles produced a porous and discontinuous network, where microparticles could not be inserted in the gel matrix without disturbing the myofibrillar gel network, as occurred in the case of nanoparticles (Yin et al., 2014b). Another nano-technological application in products of animal origin is the encapsulation of fish oil. Yang and Ciftci (2017) encapsulated fish oil in hollow solid lipid micro- and nanoparticles formed from fully hydrogenated soybean oil using atomization of supercritical carbon dioxide expanded lipid. The highest bioactive loading efficiency was $97.5 \% \mathrm{w} / \mathrm{w}$ and was obtained with a concentration of $50 \% \mathrm{w} / \mathrm{w}$, and oxidative stability of the loaded fish oil improved when compared to the free fish oil and the oxidative stability of the loaded fish oil improved when compared with the stability of the free fish oil. Thus this technique is shown to be a way of obtaining solid fish oil formulations to make handling and storage reasonable and appropriate.

Animal feeding is closely related to the meat/fish processing industry. Yausheva et al. (2016) showed that the joint utilization of iron nanoparticles and arginine raised weight gain by up to $9.2 \%$ in poultry as compared to the control, and, moreover, iron nanoparticles together with a mixture of amino acids (arginine, lysine, and methionine) provided an increase of up to $20 \%$. When applied separately, the nanoparticles and amino acids resulted in lower weight gain. Additionally, intramuscular administration of iron nanoparticles promoted an immune response that was manifested in an enhanced level of leukocytes. Iron nanoparticles have also been also 
used in combination with Lactobacillus casei as a probiotic in rainbow trout (Mohammadi and Tukmechi, 2015). Results indicated that growth parameters as weight gain, body weight gain, specific growth rate, daily growth rate, condition factor, and food conversion rate significantly increased when the diet was supplemented with iron nanoparticles $(50 \mu \mathrm{g} / \mathrm{kg})$ and Lactobacillus casei $\left(10^{8} \mathrm{cfu} / \mathrm{g}\right)$, and also intestinal bacteria counts were increased by this supplemented diet. The effect of clinoptilolite coated with silver nanoparticles on meat quality attributes of broiler chickens during frozen storage has also been evaluated (Hashemi et al., 2017). The experimental groups were fed with different diets, including supplements with $1 \%$ clinoptilolite and $1 \%$ clinoptilolite coated with 25,50 , or 75 ppm nanosilver in the basal diets, and after slaughtering they were kept for 3 or 7 days at $-17^{\circ} \mathrm{C}$. Texture profile analysis showed that nanosilver coated on clinoptilolite did not have any negative effects on texture or sensory attributes of chicken meat. The lowest value of springiness and chewiness was for the breast muscle of broilers fed with clinoptilolite coated with 25 ppm nanosilver. Adhesiveness, cohesiveness, and gumminess values were not influenced by the treatment. At all levels the addition of nanosilver coated on clinoptilolite increased water-holding capacity (WHC) in thigh muscles after 7 days of frozen storage. In conclusion, nanosilver coated on clinoptilolite can be used as a potential feed additive in broiler diet without negative implications on meat quality characteristics.

Nanoparticles have been applied in animal medicine, including diagnosis, vaccine, food administration, etc. Of special interest is their use in fish factories as treatment for antibiotic resistance. Shaalan et al. (2016) reviewed the main applications in this field, among which it is important to emphazise the use of silver, gold, zinc oxide, and titanium dioxide nanoparticles as alternatives to antimicrobials to fight microbial resistance to antibiotics; virus-like particles chitosan and polylactic-polyglycolic acid (PLGA) for drug delivery;, liposomes, immunostimulant complex, meta-nanoparticles, and gold nanoparticles for diagnosis of pathogens in fish, alone or combined with other techniques, such as loop-mediated isothermal amplification, etc. 


\subsection{ACTIVE CHITOSAN NANOPARTICLES}

Chitosan is generally recognized as safe (GRAS). It exhibits unique properties such as good biocompatibility, low toxicity, excellent biodegradability, and a broad spectrum of biological properties, including antimicrobial, antioxidant, insecticidal, and antifungal. However, chitosan shows poor solubility at pH levels above 6.5 and can only be dissolved in acidic solutions, and this limits its application as a preservative in food. This drawback could be overcome by forming chitosan nanofibers and nanoparticles that could easily be incorporated in food and have important effects on its quality. Moreover, it is worth noting that the biological properties of chitosan nanoparticles and nanofibers seem to be better than those of chitosan. Qi et al. (2004) reported enhanced antimicrobial properties of chitosan nanoparticles that were due to their small size, as well as to the quantum size effect. The preparation of chitosan nanoparticles could also improve the penetration of chitosan in tissues, which is limited by its high viscosity in solution and also by its large particle size. Gan et al. (2005) reported that submicron-sized particles could penetrate into tissues through fine capillaries, and thus the formation of chitosan nanoparticles could facilitate the incorporation of this biopolymer in foods. However, despite the bioactive potential of chitosan nanoparticles and nanofibers, the use of these particles as natural preservative agents has scarcely been reported. Chantarasataporn et al. (2014) prepared oligochitosan particles that were formed by aggregates of individual nanoparticles with an approximate size of 100-300 $\mathrm{nm}$. The oligochitosan particles were mixed with minced pork at a concentration of $0.2-0.4 \%$, and their effect on microbial quality was demonstrated throughout refrigerated storage. Thus, the incorporation of $0.4 \%$ oligochitosan in minced pork reduced growth of lactic acid bacteria (LAB) when compared with a control sample. Furthermore, the growth of total viable bacteria, Staphylococcus, and Enterobacteriaceae was significantly inhibited by the presence of either 0.2 or $0.4 \%$ oligochitosan (around $20-30 \%$ ). In addition, Salmonella sp. growth was not detected in the samples. The antibacterial effect was higher against Gram-negative bacteria than against Gram-positives. The considerable antimicrobial 
effect of these chitosan particles was probably related to the positive charge on their surface, and also to their high surface area. Moreover, the oligochitosan particles showed ability to retard the production of biogenic amines, this effect being due to the ability to produce inhibition of $L A B$ and Enterobacteriaceae. Oligochitosan nanoparticles also reduced production of secondary oxidation products and protein oxidation. This study suggests that these particles could have a considerable effect on quality and shelf life extension of processed fish or meat.

Chitosan-sodium tripolyphosphate (CH-TPP) nanoparticles have recently been incorporated in shrimp muscle to inhibit microbial growth during refrigerated storage (Chouljenko, et al., 2017a). CH-TPP nanoparticles of around $140 \mathrm{~nm}$ penetrated into meat shrimp by vacuum tumbling. The aerobic plate counts in shrimp including these nanoparticles remained constant during 24 days of storage. Furthermore, the CH-TPP nanoparticles showed an interesting antioxidant capacity, suggesting that they could be used as natural antioxidants in fish or meat products rich in polyunsaturated fatty acids. In a similar work, Chouljenko, , et al. (2017b) reported antimicrobial and antioxidant activity of similar nanoparticles incorporated in shrimp meat that was frozen for 120 days, without affecting color and texture. $\mathrm{CH}$-TPP nanoparticles have also been used to improve the quality of frozen shrimp. For example, Solval et al. (2014) used glazing solutions containing CH-TPP nanoparticles of around 180 or $234 \mathrm{~nm}$ to glaze frozen shrimp, and they observed reductions of lipid oxidation, total aerobic counts, yeasts, and molds after 30 days of storage at $-20^{\circ} \mathrm{C}$.

Owing to their properties, chitosan nanoparticles have been used as nano-sized vehicles for encapsulation of various compounds of interest to the food industry, such as essential oils. Essential oils are natural antimicrobial and antioxidant agents with interesting applications in medicine, cosmetics, and food. However, the use of essential oils in food is restricted by their potential interactions with food components, high volatility, polarity, and also negative effects on the sensory characteristics of products. Encapsulation of essential oils in chitosan 
nanocapsules could be useful to improve their solubility and stability, to mask their taste and odor, to reduce evaporation during storage, and also to allow their controlled release. Essential oil-chitosan nanoparticles could be incorporated in fish or meat products with the target of improving their overall quality and prolonging their shelf life. However, there is little information on the preservative effects of essential oils encapsulated in chitosan nanoparticles. GhaderiGhahfarokhi et al. (2017) investigated the effect of the incorporation of cinnamon essential oilloaded chitosan nanoparticles in the formulation of beef patties during cold storage. They observed that the incorporation of $0.1 \%$ cinnamon essential oil-loaded chitosan nanoparticles decreased growth of total mesophilic viable bacteria and prolonged shelf life for 4 days. Moreover, the growth of lactic acid bacteria, Enterobacteriaceae, Staphylococcus aureus, yeasts, and molds in beef patties that included the nanoparticles was lower than in the control. The incorporation of the nanoparticles also retarded lipid oxidation and improved the color and acceptability of the patties. The size of these nanoparticles was $235 \mathrm{~nm}$, and the zeta potential and polydispersity index (PDI) were $25.1 \mathrm{mV}$ and 0.33 , respectively (at $\mathrm{pH}=4.0$ ). The release mechanism of the essential oil was simulated, and a sustained release was observed, together with polymer matrix degradation in the course of time. In a similar work, the incorporation of thyme essential oil (TEO)-loaded chitosan nanoparticles (CS-NP-TEO) of around $121 \mathrm{~nm}$ in beef burgers reduced lipid oxidation and growth of Enterobacteriaceae during 8 days of storage (Ghaderi-Ghahfarokhi et al., 2016). The incorporation of CS-NP-TEO in the burgers also improved their acceptability and sensory quality. Moreover, Sotelo-Boyás et al. (2017) prepared thyme essential oil-loaded chitosan nanoparticles and nanocapsules with potent antimicrobial activity against various bacterial species, including Bacillus cereus, Staphylococcus aureus, Listeria monocytogenes, and Escherichia coli. They studied the release kinetics of the bioactive substances thymol and carvacrol, and observed that the maximum release times for thymol and carvacrol were 360 and 390 minutes, respectively. The size of these particles was $6.4 \mathrm{~nm}$ (nanoparticles) and $9.1 \mathrm{~nm}$ (nanocapsules). The interesting properties chitosan nanoparticles 
and nanocapsules loaded with thyme essential oil suggest that they could be incorporated directly in food or in polymeric active package films with the aim of prolonging shelf life.

Chitosan nanoparticles could also be useful carriers of flavors, antioxidants, minerals, vitamins, antimicrobials, or enzymes, with interesting applications in fish and meat products. The incorporation of nanoencapsulated vitamins would enhance the nutritional value of these foodstuffs. Also, encapsulation of vitamins would reduce the negative effect of high temperatures, oxygen, light, or humidity during processing and/or storage of foodstuffs, thus increasing their stability. However, no information is available about the use of nanoencapsulated vitamins in processed fish or meat products to date. Nanoencapsulated vitamins could also be introduced in feeds to reinforce the innate immunity system of fish. Nanoencapsulation would protect vitamins from harsh conditions in the intestinal tract and would permit their controlled release. Alishahi et al. (2011) used pellets including vitamin Cloaded $\mathrm{CH}$-TPP nanoparticles to feed rainbow trout, and observed that innate immunity indices were higher than those of control groups. Chitosan nanoparticles could also be useful to enhance the immunity of shrimp. For example, Rajeshkumar et al. (2009) incorporated DNAloaded chitosan nanoparticles in feed for aquaculture shrimp and reported significant protection to shrimp against white spot syndrome virus. Similar nanoparticles in feed were used by the same authors to protect farmed sea bass against Vibrio anguillarum (Rajesh Kumar et al., 2008).

\subsection{ACTIVE NANOEMULSIONS AS INGREDIENT}

Nanoemulsions are a class of emulsions (a mixture of at least two immiscible liquids wherein one phase is dispersed as small droplets within the other phase) with nanoscale droplet diameters. Specifically, oil-in-water Nanoemulsions are composed of lipid nanodroplets (with a size between 10 and $100 \mathrm{~nm}$ in diameter) dispersed in an aqueous solution (McClements, 2011). However, some authors consider a larger drop size (Wu et al., 2016; Ozogul et al., 2016; Yazgan et al., 2017). 
Droplet size is one of the most important parameters for characterizing the physical properties of an emulsion. When the droplet size decreases, the viscosity of the emulsion increases, and it becomes more stable and lighter (McClements, 2011).

The methods used to form nanoemulsions are generally divided into high-energy methods (ultrasonic devices, high-pressure homogenizers, microfluidizers) and low-energy methods (phase inversion temperature, phase inversion composition, emulsion inversion point methods) (McClements \& Rao, 2011). Among the various researchers that evaluate the application of nanoemulsions in seafood and meat products, high-energy methods, especially ultrasonication, are the ones most often employed to produce nanoemulsions (Wu et al., 2016; Ozogul et al., 2016; 2017a,b; Yazgan et al., 2017; Noori et al., 2018). Only a few research works have used other methods to obtain the corresponding nanoemulsions, such as phase inversion temperature (Moraes-Lovison et al., 2017) or the emulsion-ionic gelation technique (Wu et al., 2016). Low-energy methods are often more effective for achieve small droplet sizes, but they are more limited with regard to the types of oils and emulsifiers that can be used (McClements \& Rao, 2011).

The application for which the emulsion (micro or nano) is used in the myosystems is mainly for the preservation of meat and fish products and therefore to extent the useful life. For this, in the emulsions are added to various bioactive compounds, many of which can make undesirable odors or colorations in the final product and, being encapsulated in this way remain protected and their effect on sensory properties diminished. The application for which the emulsion (micro or nano) is used in the myosystems is mainly for the preservation of meat and fish products and therefore to extent the useful life. For this, in the emulsions are added to various bioactive compounds, many of which can make undesirable odors or colorations in the final product and, being encapsulated in this way remain protected and their effect on sensory properties diminished. 


\section{Nanoemulsions in seafood products}

Nanoemulsions are widely used in the food industry for the encapsulation of bioactives (polyphenols, carotenoids, lipids, vitamins) to improve their solubility in water, to protect them from interaction with external agents, and to enhance the bioavailability of these compounds in the human body (McClements et al., 2007). However, when they are used in direct applications in seafood or meat products, research has focused mainly on increasing the shelf life of these products. For example, Ozogul et al. (2016) studied the influence of nanoemulsions prepared with various oils on the sensory, chemical, and microbiological qualities of farmed sea bass (Dicentrarchus labrax) stored at refrigeration temperature. The results showed that the use of nanoemulsions extended the fish shelf life by two days when compared with the control, and improved the microbiological and chemical properties during the storage period. At the end of the investigation, sea bass fillets treated with nanoemulsions were described as having a neutral odor, whereas the control was characterized as having an acid and sour odor. In another study,, a sunflower oil-based nanoemulsion also improved the quality and shelf life of both sea bream (Sparus aurata) and sea bass (Dicentrarchus labrax) fillets during storage at cold temperature. In this research work, treatment with nanoemulsion reduced bacterial growth and improved the values of chemical parameters and the sensory properties of the fish samples. The influence of nanoemulsions on the preservation of Indo-Pacific king mackerel (Scomberomorus guttatus) fillets were also investigated (Joe et al., 2012), but in this study the storage temperature was 20 ${ }^{\circ} \mathrm{C}$. The application of nanoemulsion allowed an extension of shelf life of $48 \mathrm{~h}$ compared to the control and antibiotic treatment. Thus, nanoemulsion could be used to improve storage of fish at cold temperatures and in areas with poor refrigeration.

As mentioned before, in recent years, increasing interest has been shown in the use of essential oils as natural antimicrobial agents for food preservation. However, their application may be limited owing to their low solubility in water, the high volatility of some of their constituents, 
and their strong flavor and odor, which could affect the sensory properties of the food product. One strategy used to overcome these disadvantages is the encapsulation of essential oils. Various works have demonstrated that encapsulation in nanoemulsions improves the bioactivity of essential oils because it increases diffusion through biological membranes, owing to their nanoscale and lipophilic nature (Donsì et al., 2011). Nanoemulsions could also protect essential oils against evaporation, oxidation, or thermal degradation, and increase their stability, allowing a reduction in the dose of essential oils required to ensure their bioactivity and minimize their impact on the sensory properties of food (Donsì et al., 2011). Furthermore, nanoemulsions can be considered as self-preserving antimicrobials because water is bound by their structure, restricting the access of microorganisms to water (Al-Adham et al., 2000). In this context, Wu et al. (2016) prepared coatings with emulsions and nanoemulsions based on chitosan particles loaded with citrus essential oil, and investigated the effect of these coatings on the shelf life of silver pomfret fillet stored under refrigeration. Treatment with nanoemulsions was more effective than conventional emulsions, extending the durability of fish samples from 12 days to 16 days, while the control sample had a shelf life of only 8 days. The nanoemulsion coating was effective in inhibiting the growth of microorganisms and changes in the chemical properties of the silver pomfret. Furthermore, the fish treated with a nanoemulsion coating had the highest sensory scores at the end of storage. On the other hand, jujube gum-based nanoemulsions containing nettle essential oil as edible coatings were used to preserve beluga sturgeon fillets during storage under refrigeration. Physical, chemical, microbiological, and sensory characteristics of the fish samples were evaluated. The results of this work showed that the shelf life of the fish samples was extended by the use of the corresponding edible coating. A coating formulated with $12 \%$ jujube gum and $3.5 \%$ nettle essential oil allowed the lowest microbial growth, weight and cooking losses, $\mathrm{pH}$ changes, and sensory deterioration in beluga sturgeon (Gharibzahedia \& Mohammadnabiba, 2017). Two other studies evaluated the effect of the application of nanoemulsions on the shelf life of rainbow trout fillets during cold storage. 
Shadman et al. (2017) prepared sunflower oil nanoemulsions containing various concentrations of Zataria multiflora Boiss essential oils, and determined the chemical and sensory properties of the fish samples. Ozogul et al. (2017b) used nanoemulsions based on different herb oils (rosemary, laurel, thyme, and sage) and evaluated the microbiological quality, chemical properties, and sensory properties (raw and cooked) of the fish fillets. Both studies found that the quality of the fish fillets was improved by using nanoemulsions.

The lipid oxidation process can also be retarded by the use of nanoemulsions. Ozogul et al. (2017a) found that the application of nanoemulsion in combination with vacuum packing allowed a lower loss of polyunsaturated fatty acid (PUFA) content during storage of sea bass fillets compared to the control. This could be attributed to the effect of the vacuum and nanoemulsion on prevention of lipid oxidation. In some of the investigations that studied the preservative effect of nanoemulsions in seafood products, the effect on lipid peroxidation was also evaluated. Some of these works found that the use of nanoemulsion also retarded the lipid oxidation process in fish samples (Yazgan et al., 2017; Gharibzahedia \& Mohammadnabiba, 2017; Shadman et al., 2017).

Nanoemulsions could also be used in the aquaculture industry. The main used is for avoid infection in fish. Mishra et al. (2014) highlights the therapeutic potential of neem nanoemulsion against Pseudomonas aeruginosa infection in the freshwater fish Labeo rohita. The nanoemulsion formulation reduced the toxicity effect of neem oil, leading to a treatment with negligible toxicity for the fish. The effect of neem nanoemulsion for controlling $P$. aeruginosa infection in L. rohita was similar to that of the commercial antibiotic kanamycin.

\section{Nanoemulsions in meat and poultry products}

In meat and poultry products, the researches have been carried out using essential oils encapsulate in nanoemulsions to preserve the self-life. Noori et al. (2018) compared the effect of conventional emulsion and nanoemulsion-based coatings containing ginger essential oil on 
the safety and quality of chicken breast fillets during storage under refrigeration. Similarly, to the above-mentioned research ( $\mathrm{Wu}$ et al., 2016), the nanoemulsions allowed a better preservation of foods compared to the emulsions. A nanoemulsion-based edible sodium caseinate coating containing $6 \%$ of ginger essential oil improved the shelf life of chicken breast. The positive effect on appearance was again another important benefit of application of the nanoemulsion. Samples coated with ginger essential oil nanoemulsions showed lower color difference in comparison with the fresh chicken fillets than those coated with emulsions. Furthermore, when nanoemulsion was used in the formulation of the coating, the corresponding sample showed the highest total score during storage. However, in this study the nanoemulsion treatment did not have a significant effect on lipid oxidation of chicken fillets. On the other hand, Moraes-Lovison et al. (2017) developed nanoemulsions encapsulating various amounts of oregano (Origanum vulgare) essential oil. In this research, the nanoencapsulation of essential oil did not significantly improve its antimicrobial properties as initially expected. However, when the nanoemulsion was incorporated in chicken pâté previously inoculated with Escherichia coli, the contamination on the last day of analysis was significantly lower than with direct oregano essential oil treatments.

The results of all of these studies confirm the potential utility of essential oil nanoemulsion for improving the shelf life of poultry meat or fish products.

\subsection{NANOLIPOSOMES}

Liposomes are vesicles of spherical shape surrounded by one or more phospholipid bilayers that are used for encapsulation and delivery of a diversity of hydrophilic and hydrophobic substances. In the field of the food industry, liposomes can be used as flavor encapsulation maskers to hide bitter tastes, and also to enrich functional foods with healthy and bioactive compounds, such as antioxidants, vitamins, minerals, peptides and enzymes, that could be useful for disease prevention (Mozafari et al., 2008; Singh, 2016). Liposomal encapsulation improves chemical and 
physical stability of the active compounds, prevents their interactions with other food constituents, and favors their targeted delivery inside the organism (da Silva Malheiros et al., 2010). Liposomes are produced from the nanometer to the micrometer size, and their structural and physicochemical properties depend strongly on the method of preparation and lipid composition (Akbarzadeh et al., 2013).

A variety of lipids can be used for liposome preparation. Specifically concerning liposomes for the food industry, the high content in polyunsaturated fatty acids and residual tocopherol, both being naturally present in the soy lecithin or semi-purified phosphatidylcholine, provide a beneficial role in lipid metabolism and high antioxidant activity (Taladrid et al., 2017). The use of natural phospholipids reduces considerably manufacturing costs and meets food legislation (Laye et al., 2008). However, the unsaturated fatty acid composition decreases the bilayer thermal stability and confers higher susceptibility to lipid oxidation (Wang and Wang, 2008), causing rancidity and shelf life reduction when included in a food system. The encapsulation of antioxidant compounds in liposomes contributes to prevent lipid oxidation. However, liposomal stability, encapsulation efficiency and particle properties will depend on the type of antioxidant and its concentration (Tan et al., 2013).

Liposomal suspensions can be stable for several weeks, however they usually become unstable by aggregation, oxidation and/or hydrolysis, that could lead to sedimentation and drug leakage (Sharma and Sharma, 1997). Freezing (Chen et al., 2010), freeze-drying (Sebaaly et al., 2016) or spray-drying (Gültekin-Özgüven et al., 2016) have been proposed to enhance liposomal stability during long-term storage. However, they normally induce an increase in the particle size, especially the freezing method. Lyophilization may cause damage in the vesicle membrane by the production of ice crystals followed by aggregation during freezing and subsequent desiccation, as well as phase transition changes during liposome reconstitution (Chen et al., 2010). Polyalcohols and carbohydrates have been used to prevent freezing- or freeze-drying- 
induced damage of the bilayers (Stark et al., 2010). Various natural extracts with antioxidant capacity (pomegranate peel extract, shrimp lipid extract and collagen hydrolysate) were successfully entrapped in soy phosphatidylcholine nanoliposomes together with glycerol. Freeze-drying caused the particle size to increase from $75.7-81.0 \mathrm{~nm}$ up to $199-283 \mathrm{~nm}$, however a considerable amount still remained near the original nanoscale (Marín et al., 2018a). Noticeable changes in the liposome bilayer caused by the freeze-drying process and long-term storage were found to depend on the encapsulation efficiency and the type of the loaded compound (Marín et al., 2018b). Of particular relevance to the food industry is the fact that the incorporation of liposomes in the dry state makes easier the adjusting of the moisture content in the formulation of meat and fish restructured products and pâtés. This effect is highly advantageous for texture optimization in functional foods with a low or intermediate moisture level. Besides their bioactive potential, liposomes can also be used in the food industry for a variety of technological purposes, providing controlled release of antimicrobials and antioxidants for food preservation during storage, improving industrial processes, or avoiding health risks.

Liposomes are vesicles of spherical shape surrounded by one or more phospholipid bilayers that are used for encapsulation and delivery of a diversity of hydrophilic and hydrophobic substances. In the field of the food industry, liposomes can be used as flavor encapsulation maskers to hide bitter tastes, and also to enrich functional foods with healthy and bioactive compounds, such as antioxidants, vitamins, minerals, peptides and enzymes, that could be useful for disease prevention (Mozafari et al., 2008; Singh, 2016). Liposomal encapsulation improves chemical and physical stability of the active compounds, prevents their interactions with other food constituents, and favors their targeted delivery inside the organism (da Silva Malheiros et al., 2010). Liposomes are produced from the nanometer to the micrometer size, and their structural and physicochemical properties depend strongly on the method of preparation and lipid composition (Akbarzadeh et al., 2013). 
A variety of lipids can be used for liposome preparation. Specifically concerning liposomes for the food industry, the high content in polyunsaturated fatty acids and residual tocopherol, both being naturally present in the soy lecithin or semi-purified phosphatidylcholine, provide a beneficial role in lipid metabolism and high antioxidant activity (Taladrid et al., 2017). The use of natural phospholipids reduces considerably manufacturing costs and meets food legislation (Laye et al., 2008). However, the unsaturated fatty acid composition decreases the bilayer thermal stability and confers higher susceptibility to lipid oxidation (Wang and Wang, 2008), causing rancidity and shelf life reduction when included in a food system. The encapsulation of antioxidant compounds in liposomes contributes to prevent lipid oxidation. However, liposomal stability, encapsulation efficiency and particle properties will depend on the type of antioxidant and its concentration (Tan et al., 2013).

Liposomal suspensions can be stable for several weeks, however they usually become unstable by aggregation, oxidation and/or hydrolysis, that could lead to sedimentation and drug leakage (Sharma and Sharma, 1997). Freezing (Chen et al., 2010), freeze-drying (Sebaaly et al., 2016) or spray-drying (Gültekin-Özgüven et al., 2016) have been proposed to enhance liposomal stability during long-term storage. However, they normally induce an increase in the particle size, especially the freezing method. Lyophilization may cause damage in the vesicle membrane by the production of ice crystals followed by aggregation during freezing and subsequent desiccation, as well as phase transition changes during liposome reconstitution (Chen et al., 2010). Polyalcohols and carbohydrates have been used to prevent freezing- or freeze-dryinginduced damage of the bilayers (Stark et al., 2010). Various natural extracts with antioxidant capacity (pomegranate peel extract, shrimp lipid extract and collagen hydrolysate) were successfully entrapped in soy phosphatidylcholine nanoliposomes together with glycerol. Freeze-drying caused the particle size to increase from $75.7-81.0 \mathrm{~nm}$ up to $199-283 \mathrm{~nm}$, however a considerable amount still remained near the original nanoscale (Marín et al., 2018a). Noticeable changes in the liposome bilayer caused by the freeze-drying process and long-term 
storage were found to depend on the encapsulation efficiency and the type of the loaded compound (Marín et al., 2018b). Of particular relevance to the food industry is the fact that the incorporation of liposomes in the dry state makes easier the adjusting of the moisture content in the formulation of meat and fish restructured products and pâtés. This effect is highly advantageous for texture optimization in functional foods with a low or intermediate moisture level. Besides their bioactive potential, liposomes can also be used in the food industry for a variety of technological purposes, providing controlled release of antimicrobials and antioxidants for food preservation during storage, improving industrial processes, or avoiding health risks. Furthermore, the liposomes included in caseinate films has been observed that can be rise the adhesive and mucoadhesive properties facilitating the oral dispersibility of liposomes (Montero et al., 2019),

\section{Nanoliposomes in meat processing}

There is a growing demand for healthy and safe food products with additional benefits beyond basic nutrition. In this context, the fortification of meat pâté with iron salts encapsulated in liposomes has been proposed as a dietary strategy to prevent anemia in young iron-deficient women (Navas-Carretero et al., 2009). In a previous study in rats fed with fortified meat pâté it was observed that iron bioavailability from ferrous gluconate was similar to that of a commercial liposomal-encapsulated ferric pyrophosphate preparation (Navas-Carretero et al., 2007). Meatbased products are rich in proteins with high biological value, vitamins, minerals, and other bioactive compounds, such as taurine. The main negative aspect is their high content in cholesterol and saturated fatty acids, especially in red meat, which has been largely related to cardiovascular risk. The nutritional quality of pork meat was improved by the incorporation of polyunsaturated omega-3 fatty acids ( $42 \%$ eicosapentaenoic and $16 \%$ docosahexaenoic) encapsulated in nanoliposomes (Ojha et al., 2017). Diffusion of the encapsulated oil into the 
pork meat slices was enhanced by ultrasound treatment to overcome the low rates of diffusion into the solid food matrix.

Lipid oxidation may negatively affect the shelf life and quality of meat products, particularly in the case of ground meat. There is a growing tendency to replace synthetic antioxidants with other natural derivatives from animals or plants, which, owing to their non-toxic nature, provide greater safety for consumer health. Nanoliposome technology was proposed to mask the fishy smell of marine fish skin peptides (FSP) incorporated as a natural antioxidant in a pork patty model (Bai et al., 2017). These authors found that the FSP inhibited the lipid oxidation of the patties during refrigerated storage; however, the TBARS values were significantly higher than in the control sample. Furthermore, the amount of volatile basic nitrogen was not efficiently reduced by peptide encapsulation. As a positive aspect, patties with liposomes showed lower cooking losses than patties treated with conventional Butylate hydroxy Anisole (BHA) antioxidant. The authors concluded that liposomes made from lecithin were not good candidates for encapsulation of antioxidant peptides to be applied as meat preservatives.

Meat biopreservation by using bacteriocins, which are heat-stable antimicrobial peptides of bacterial origin, represents an innovative approach to the control of spoilage and pathogenic microorganisms. In particular, nisin and pediocin are very interesting in terms of food safety since they are generally recognized as safe substances (GRAS). However, bacteriocins may be less effective in meats because they are degraded by proteases, and their adsorption to sensitive microorganisms could be influenced by the presence of various salts. Encapsulation in nanoliposomes has been reported to provide bacteriocins with higher antimicrobial efficiency and stability (da Silva Malheiros et al., 2010). Pediocin AcH was encapsulated in multilamellar vesicles of phosphatidylcholine to afford protection and more even distribution of this antimicrobial in heated slurries of beef tallow and beef muscle (Degnan and Luchansky, 1992). The results of this work showed increases of around $28 \%$ in the recovery of pediocin activity 
when pediocin was encapsulated as compared to the free form. Furthermore, dairy-based slurries showed greater enhancement of pediocin activity than meat-based slurries (Degnan et al., 1993). Besides the action of proteases, nisin could also be inactivated by enzymatic reaction with glutathione, a low molecular weight sulfur compound present in meat tissues. To maximize the activity of nisin in meat during cooking and storage, it was encapsulated in dipalmitoylphosphatidylcholine liposomes, which were prepared without the use of organic solvents (Boualem et al., 2013). Encapsulated nisin incubated with raw beef did not produce any antimicrobial effect; however, the release of nisin after cooking was found to form clear inhibition zones. It was concluded that nisin was protected inside the vesicle and was released from the melted liposomes during cooking.

Lytic bacteriophages have received a great deal of attention as natural food preservatives with GRAS status, for example to reduce the prevalence of $E$. coli $\mathrm{O} 157: \mathrm{H7}$ and other food pathogens that could be present in beef products. To avoid their antibacterial activity in the presence of enzymes, evaporite materials and acidic compounds in foods, phages have also been encapsulated in soy lecithin/cholesterol liposomes with an encapsulation efficiency of $58 \%$ and mean particle size of $150 \mathrm{~nm}$ (Cui et al., 2017a). The phage-loaded liposomes were embedded in a chitosan film to provide increased stability. The liposomal encapsulation increased the phages stability, and the resulting complex film applied on beef pieces showed activity against E. coli $0157: \mathrm{H7}$. The film did not change the surface color of beef compared to that of the control group, and it scored an overall better sensory evaluation value.

Essential oils have also been applied recently in meat processing and preservation, being also considered generally as GRAS substances. They present notable limitations owing to their inherent volatility, which may decrease their antimicrobial activities and impair the sensory quality of the meat. Furthermore, the adsorption of active components onto fat globules in meat products may also produce a negative effect on the essential oil antimicrobial activity. In this 
respect, thyme oil encapsulated in nanoliposomes of soy lecithin and cholesterol was reported to be more efficient in controlling the growth of S. enteritidis in cchicken when compared with free thyme oil, without negatively affecting the sensory quality (Cui et al., 2017b). In a recent work, cinnamon essential oil was encapsulated in beta-cyclodextrin proteoliposomes of nanometric size which were fixed in a support of poly(ethylene oxide) nanofibers as an antimicrobial packaging system (Lin et al., 2017). The complex nanofibers significantly extended the shelf life of beef stored at $4{ }^{\circ} \mathrm{C}$, with a significant reduction in the $B$. cereus population and no impact on the meat sensory properties.

Polyphenols with high antioxidant activity can also be useful to decrease the formation of harmful substances, such as heterocyclic aromatic amines (HAA), in fish and meat cooked at temperatures above $150{ }^{\circ} \mathrm{C}$. These antioxidants may lose their efficacy due to excessive high temperatures or to oxygen or light exposition, so liposomal entrapment could be a way of improving their stability and favoring controlled delivery. The functionality of grape seed extract (GSE) encapsulated in soy lecithin nanoliposomes to prevent HAA formation in fried beef patties after marination was investigated (Natale et al., 2014). The encapsulation of the GSE in liposomes did not significantly prevent the formation of HAA compared to the use of free GSE. The authors hypothesized that the very small size of the particles caused diffusion into the interior of the meat, being less effective at inhibiting the reaction of HAA formation that occurs mostly at the surface.

Liposome technology has also been applied for meat tenderization purposes. Calcium encapsulated in egg phosphatidylcholine liposomes was injected into rabbit before slaughter to accelerate the meat aging process (Kim et al., 2006). Liposome encapsulation of calcium was developed as a method for increasing the $\mathrm{Ca}^{2+}$ concentration in the muscle through the blood vessels without causing animal shock and death. Liposomes produced with a $2 \mathrm{M} \mathrm{Ca}^{2+}$ concentration and size of $0.25-1.8 \mu \mathrm{m}$ were found suitable for injection into rabbit before 
slaughtering to produce effective meat tenderization without causing any physical shock or contamination. Bromelain can also be used as a tenderizing agent in meat processing. Soy phosphatidylcholine liposomes with high particle stability have been proposed as a potential tool to increase the availability of bromelain

\section{Nanoliposomes in fish products}

Surimi gels could be an excellent food model for enrichment with bioactive ingredients. However, the biological activities may be hampered during the thermal gelation due to excessive interactions with the muscle protein or degradation upon heating (Montero et al., 2005). Furthermore, the encapsulation using highly unsaturated phospholipids contribute greatly to enrich the healthy design of the functional surimi gels. Freeze-dried soy phosphatidylcholine liposomes entrapping various natural antioxidants (pomegranate peel extract, collagen hydrolysate, shrimp lipid extract) were shown to decrease the gel strength and to improve the stability of surimi squid gels during long-term frozen storage. The entrapped extracts, however, were unable to decrease lipid oxidation in the gel matrix. The addition of liposomes causing weaker gel matrices by disturbing protein-protein interactions was found to enhance protein digestibility (Marín et al., 2018b). The addition of various liposomal preparations (fresh, frozenthawed, spray-dried and freeze-dried) to salt-ground fish mince was found to increase the water holding capacity of the solubilized protein, but they interfered with the thermal aggregation ability (Marín et al., 2018c). The liposome hydration state (wet or dry), rather than the surface charge or vesicle size, was the most important factor influencing the thermal gelation profile. In figure 1 you can see the appearance of a salt ground hake mince without and with the addition of fresh liposomal solution, as well as the consequent thermal gels obtained. The difference in color attributed to the liposomes can be observed and the differences in the gels decrease after the thermal treatment. 


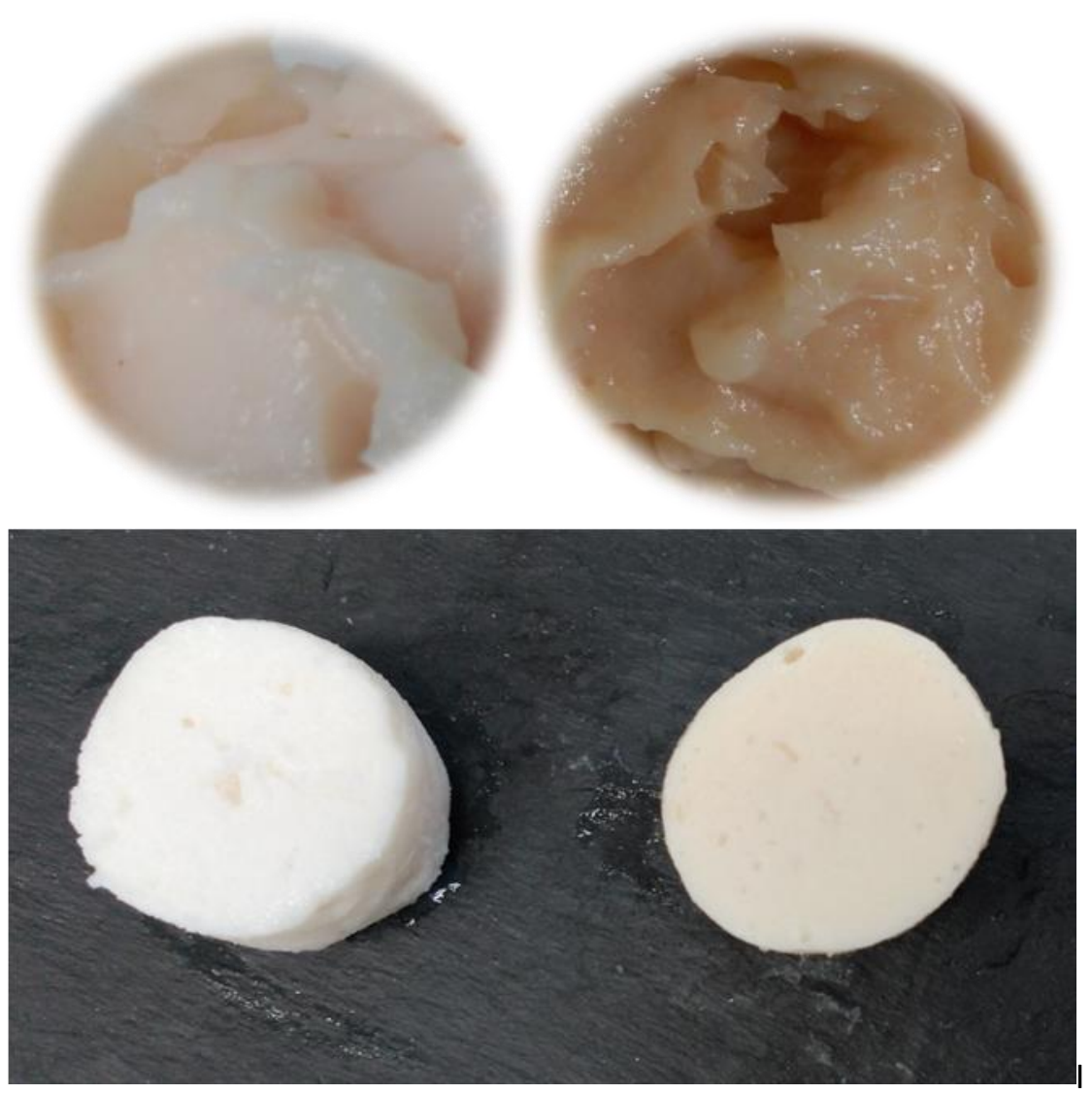

Figure 1.- Salt ground hake muscle without A) and B)with liposomes, and their respective thermal gels $C$ and $D$ )

The use of lipids for protein gel reinforcement has been previously reported. It has been suggested that lipid droplets do not interact with proteins in the gel network, but act as filler in the matrix pores (Mor et al., 1999). In gelatin gel systems, liposomes located into the pores of the matrix improved the release of the bioactive compounds during storage (Mufamadi et al., 2011). Nanoliposomes containing Angiotensin converting enzyme (ACE)-inhibitory peptides with an encapsulation efficiency of $53 \%$ were included in cold-induced fish gelatin gels without hindering the thermal stability and viscoelastic properties. Furthermore, the liposomal encapsulation did not alter the ACE-inhibitory activity of the peptide fraction, thus keeping the bioactive potential of the complex gelatin gel (Mosquera et al., 2016). 
A functional edible wrapping based on cooked shrimp protein was developed by including $\varepsilon$ polylysine-adsorbed liposomes entrapping antioxidant and ACE-inhibitory peptides (Alemán et al., 2016). The complexation with $\varepsilon$-polylysine increased the liposome size from $164 \mathrm{~nm}$ to 216 $\mathrm{nm}$ and was effective to increase the liposome stability and the antimicrobial capacity. The complex wrapping, which has desirable sensory properties and activity against E. coli, L. monocytogenes, $Y$. enterocolitica and $S$. aureus, could be used to design a variety of functional foods, without the requirement for product reformulation or restructuring. Figure 2 shows the aspect of this film as well as an example of functional food product.
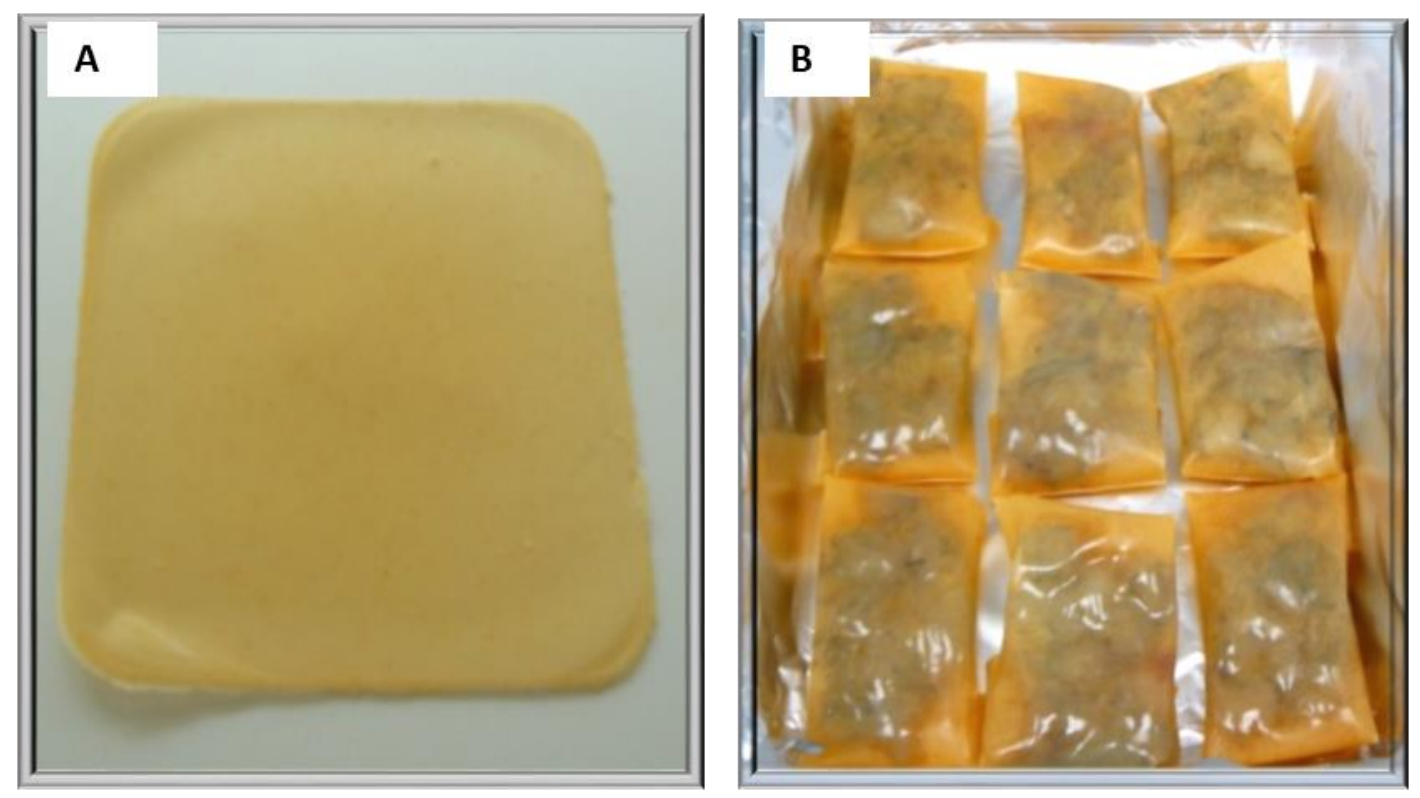

Figure 2.- A), myofibrillar shrimp protein film containing phosphatidylcholine soya nanoliposomes loaded with bioactive peptides adsorbed with polylysine B) raw shrimp tails wrapped with this film as a culinary preparation.

Natural polyphenol-rich plant extracts and essential oils have also been used to extend the shelf life of fish products, due to their antimicrobial and antioxidant potential. Thyme extract encapsulated in liposomes and incorporated in minced silver carp inhibited the growth of mesophilic and psychrotrophic bacteria during chilled storage compared to samples containing 
the unencapsulated extract (Javadian et al., 2017). Liposomes loaded with $0.5 \%$ thyme extract were also able to reduce the population of $E$. coli $0157: \mathrm{H} 7$. The overall quality of the minced fish was significantly improved by reducing the peroxide value, the total volatile basic nitrogen, and the microbial load during storage.

The use of liposomes based on marine phospholipids has gained attention in the aquaculture field. The main problems faced by aquaculture are the survival of fish larvae and the difficulty of supplying live prey organisms for larva feeding. Liposomes with high content in long-chain $n-3$ PUFA (eicosapentaenoic and docosahexaenoic fatty acids) can provide an adequate lipid profile to prey organisms, with subsequent benefits for the survival, growth, and development of fish larvae (Henna Lu et al., 2011). Owing to the ability to encapsulate lipids and water soluble components, many studies have dealt with the successful use of liposomes to enrich live prey, such as Artemia nauplii, with a number of nutrients, namely vitamins A and C (Monroig et al., 2007), free amino acids (Saavedra et al., 2008), and essential fatty acids (Monroig et al., 2006).

\section{APPLICATIONS IN PACKAGES FOR MEAT AND FISH}

One important function of packaging, , is to retard food product deterioration, improving quality and safety and prolonging shelf life (Lopez-Rubio et al., 2004). This becomes especially important in the case of fish and meat due to their short shelf life. The current trend in food packaging is the design of active packages that interact with the food product improving its quality and safety (Angiolillo et al., 2017; Lopez-Rubio et al., 2004). The other tendency in the packaging sector is the development of intelligent packages, which check the storage conditions and the quality of the packaged food item (Angiolillo et al., 2017). The unique physicochemical properties of materials at the nanoscale provide high potential for the development of active or intelligent packages, which are two of the main current applications of nanotechnology in food packaging. Additionally, improvement of the physicochemical properties of polymeric materials and 
enhancement of the use of biodegradable packages are also areas in which nanotechnology is being applied (Angiolillo et al., 2017; Sanchez-Garcia et al., 2010; Sharma et al., 2017). In spite of this, most of the work in this topic is related to research rather and commercial applications are scarce (Angiolillo et al., 2017). These three main applications of nanotechnology in the food packaging sector are discussed below:

\section{Improvement of physicochemical performance of food packaging materials}

Among the variety of materials available for food packaging, polymers prevail over glass, tinplate, or paper because of several advantageous properties such as low cost, light weight, ease of printing, microwaveability, thermosealability, flexibility, etc. Furthermore, the variety of existing polymers and processing technologies provides high versatility regarding package physical properties, shape, size, etc. Indeed, there are a number of packaging materials with appropriate physical properties such as gas, vapor, and aroma barrier, mechanical properties, transparency, etc., that can be adjusted to specific applications, so almost all types of foods can be found packed in polymeric packages. Thanks to this, meat and fish can be packed in bags or trays under vacuum or modified atmosphere, preserving the desired gas headspace composition. However, in spite of the improved properties, the gas barrier properties of glass and tinplate are still superior, so nanotechnology can improve the performance of conventional polymers. Another drawback of polymeric materials is that they are made from non-renewable resources (petroleum), which are generally not biodegradable and accumulate in the environment. Furthermore, recycling is not easy, as they are commonly made of multilayer and composite structures. Consequently, research on compostable or biodegradable biopolymers has gained great interest in recent years. For most biodegradable polymers it is necessary to improve some of their properties, such as gas barrier characteristics and mechanical properties. Reinforcement of these biodegradable polymers to prepare nanocomposites has already been proven to be an effective way of enhancing these properties concurrently (Gómez-Estaca et al., 
2016; Sanchez-Garcia et al., 2010: Echeverría et al., 2016, 2018).Nanocomposites can be defined as materials having filler with at least one dimension smaller than $100 \mathrm{~nm}$ (Arora \& Padua, 2010). This new generation of composites exhibits significant improvements in modulus, dimensional stability, and solvent or gas resistance with respect to pristine polymer (Sorrentino et al., 2007). Among the nanomaterials incorporated in food packaging nanocomposites, it is worth mentioning cellulose fibers and nanowhiskers, carbon nanotubes and nanofibers, clays and silicate nanoplatelets, starch nanocrystals, and chitin/chitosan nanoparticles, for mechanical, thermal stability, and/or barrier performance improvements (Sanchez-Garcia et al., 2010; Sharma et al., 2017). Other developments do not necessarily include compositing of the polymers and nanomaterials, as multilayer structures can also be designed in which one of the layers is on the nanoscale. One example would be the coating of a very thin layer of nanocellulose onto conventional polymeric films, or the application of ultrathin metal coatings (Gokularaman et al., 2017; Li et al., 2015). Thanks to the application of nanotechnology to improve the physicochemical performance of packaging materials, the quality of packed meat or fish will be improved. In this manner, packages with better mechanical, thermal, and barrier properties will protect the packed product more efficiently. Furthermore, packaging materials from alternative eco-friendly polymers will be available for these packaging applications. As an example, Lloret et al. (2016) developed a polyamide/nanoclay nanocomposite that preserved dry-cured ham as efficiently as a high-barrier commercial polymer, whereas unmodified polyamide film failed to preserve product color during the 90-day refrigerated storage period as a consequence of its higher oxygen transmission rate.

\section{Development of active packages}

The traditional concept of the food package as a passive barrier to protect food from the effect of the environment has evolved to the active packaging concept, as a result of the novel consumer trends toward minimally processed and convenient food products with a prolonged 
shelf life. Therefore, active packages interact with the packed product or its surrounding atmosphere with a beneficial outcome. There are a number of active packages with applications in many different food products (Lopez-Rubio et al., 2004), but oxygen scavengers, antioxidant, and especially antimicrobial active packages stand out as the most important developments for poultry, meat, and fish owing to their inherent characteristics (Gómez-Estaca et al., 2014; LópezCarballo et al., 2012; McMillin, 2017). Focusing on nanotechnological applications, developments can consist in nanostructuration of the active compound that is incorporated into the active packaging system (film, pad, tray, etc.) or incorporation of a non-nanostructured active compound into a nanocomposite whose properties allow modulation of its release, as compared to unmodified polymers. Encheverría et al (2016) observed that the addition of clove essential oil increased the degree of exfoliation of the clay in the isolated soybean protein film, observing that exfoliated and interspersed nanostructures coexist in these materials.

With regard to oxygen scavengers based on nanotechnology, Xiao-e et al. (2004) developed nanocrystalline $\mathrm{TiO}_{2}$ films with the ability to deoxygenate a closed environment under continuous UV irradiation. However, there are no applications of this packaging material on myosystems, which would be especially interesting for poultry or white fish.

The most studied nanoparticles used as antimicrobials are metals ( $\mathrm{Ag}, \mathrm{Au}, \mathrm{Zn}, \mathrm{Cu}$ ) or metal oxides $\left(\mathrm{TiO}_{2}, \mathrm{ZnO}, \mathrm{SiO}_{2}, \mathrm{MgO}\right.$ ) (Angiolillo et al., 2017; Espitia et al., 2016; Sharma et al., 2017). Among them, silver nanoparticles are one of the most studied, and various mechanisms have been proposed to throw light upon their antimicrobial property: adhesion to the cell surface with consequent alteration of membrane electric charges; structural modification of membrane lipopolysaccharides; DNA damage; or binding to groups such as sulfur, oxygen, or nitrogen (Angiolillo et al., 2017; Rai et al., 2009). Most of the processes for the synthesis of silver nanoparticles involve the formation of colloidal nanoparticles or their incorporation in other materials (Rai et al., 2009; Sharma et al., 2010). Nanosilver-based active packages have proven 
their antimicrobial effectiveness in vivo. As an example, Azlin-Hasim et al. (2016) developed composite $\mathrm{Ag} / \mathrm{PVC}$ (polyvinylchoride) films that exerted antimicrobial action when applied to chicken breast fillets. Fernández et al. (2010) developed absorbent pads by silver nanotechnology, reducing spoilage-related microflora of packed beef meat. Prior to commercialization of silver-based food packaging systems, it is mandatory to reduce the migration below the limit allowed $(0.05 \mathrm{mg} \mathrm{Ag} / \mathrm{kg}$ food). Indeed, particulate migration into foods does not occur in many applications, so the potential risk to consumers is reduced (Wyser et al., 2016). Other metal-based nanoparticles have also shown good results. Low-density polyethylene films loaded with $\mathrm{TiO}_{2}$ and $\mathrm{ZnO}$ nanoparticles showed antimicrobial activity against E. coli 0157:H7 in calf minced meat (Marcous et al., 2017). Zinc oxide has also shown good results for packing pork or poultry (Akbar \& Anal, 2014; Suo et al., 2016). In spite of these positive results, overall toxicological data indicate that more research is required before metal-based nanoparticles can be tagged as toxic or safe (Sharma et al., 2017). In any case, application of these kinds of antimicrobials seems to be restricted to active packaging applications, which may make it possible to retard the release and adjust it to an effective antimicrobial dose at the food surface, where microbes grow, without reaching a toxic dose.

Apart from metal and metal oxide nanoparticles, nanocomposites including nanoclays have been developed in order to retard the release of the antimicrobials incorporated. This is the case with a bentonite nanoclay/EVOH composite developed to retard the release of an added volatile antimicrobial (carvacrol), without significant drawbacks affecting the other physicochemical properties studied thanks to the good dispersion of bentonite (Cerisuelo et al., 2012). This material may be applied to fresh fish preservation, as a food packaging material based on an active PP/EVOH/PP film in which carvacrol was added showed its effectiveness as an antimicrobial when applied on refrigerated salmon (Cerisuelo et al., 2013). In another work, Alboofetileh et al. (2016) developed an alginate/clay nanocomposite incorporating marjoram essential oil and showed a good antilisterial effect in vitro, as well as a delay of $L$. 
monocytogenes, total viable count, and psychrotrophic growth when applied to the preservation of rainbow trout slices at refrigeration temperature. In another work, an active nanocomposite film based on soy proteins and montmorillonite incorporated with clove essential oil exerted antimicrobial and antioxidant activity on refrigerated bluefin tuna fillets, without diffusion of the clay's own metals to the muscle (Echeverría et al 2018) showing that the presence of clay facilitates the controlled release of clove essential oil, while the presence of clove essential oil seems to promote the exfoliation of clay (Echeverría 2016). Figure 3 shows the looks of the active nanocomposite film as well as the appearance of the tuna fillet coated by this film. Regarding antioxidant activity, Busolo and Lagaron (2015) developed polyethylene films based on a resveratrol-containing clay that delayed lipid oxidation of fresh meat packed in direct contact with it.
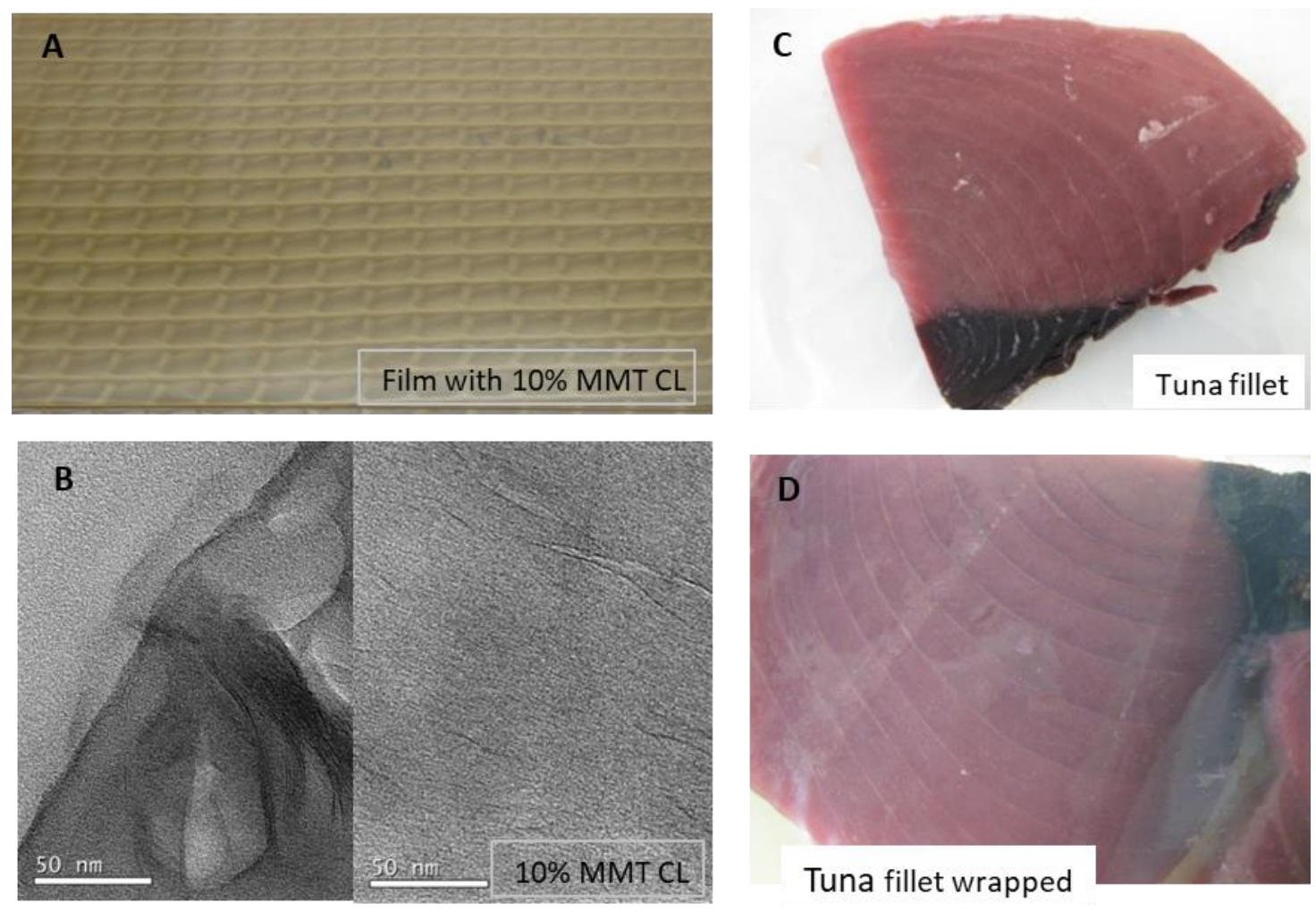

Figure 3.- A) Film made with soy protein, clove essential oil and montmorillonite nanocomposite. B) TEM of nanocomposite film based on soy protein isolate with $10 \%$ 
of montmorillonite and $0,5 \mathrm{ml}$ clove essential oil. C) Appearance of tuna fillet D) Appearance of a tuna fillet (Tumnus thymus) coated with this film.

\section{Development of intelligent packages}

Intelligent packages are those capable of interacting with the packed food, recognizing any of their characteristics, and applying various mechanisms to record and convey information, with the final objective of boosting consumer information about quality and safety (Sharma et al., 2017). There are a number of intelligent packaging developments, including indicators (gases, temperature, freshness), data carriers (barcodes, radio-frequency identification systems), and sensors, these last being the ones with the highest potential for future developments (Ghaani et al., 2016). A sensor is a device or system with control and processing electronics, an interconnection network, and software (Patel and Beveridge, 2003). Nanotechnological applications are mostly important in the first part of the system, that is, the receptor, which is the sensing part of the sensor and is represented by a sampling area where the surface chemistry occurs. In this context, the unique chemical and electro-optical properties of nanoscale particles made them of special interest (Bouwmeester et al., 2009). The main nanotechnological developments for meat and fish include $\mathrm{O}_{2}$ and $\mathrm{CO}_{2}$ indicators/sensors, detection of spoilage and pathogenic microorganisms, freshness indicators, and time temperature indicators (Sharma et al., 2017).

Many food products, including fish and meat, are preserved under vacuum or modified atmosphere with limited oxygen access. In these specific cases, an $\mathrm{O}_{2}$ indicator will give the consumer information about the quality of the food, as inefficiency in the packaging system or damage of the package would induce changes in the headspace gas composition. With regard to the application of nanotechnology, developments based on $\mathrm{TiO}_{2}$ or $\mathrm{SnO}_{2}$ nanoparticles for oxygen detection have been described (Lee et al., 2005; Mills \& Hazafy, 2009). Carbon dioxide is 
also considered as an active packaging gas because high levels reduce the metabolic rates of microbes. Therefore, detection of a decrease in its original concentration will give the consumer valuable information about the quality of the packed meat or fishery product (Puligundla et al., 2012). There are various systems for $\mathrm{CO}_{2}$ determination for food packaging applications (Puligundla et al., 2012; Sharma et al., 2017), and future developments applying nanotechnology should be expected.

Another group of intelligent packaging systems is bacteria detection systems, notably those consisting of antibodies conjugated to nanomaterials such as quantum dots. This system is based on antibody antigen interaction, boosted by the unique optical and electrical characteristics of nanoparticles in conjunction with other properties such as spacious and simply functionalized surfaces (Sharma et al., 2017). An example of a quantum dot-based system with potential applications in meat packaging was developed by Yang and Li (2006). This system was able to detect the presence of $E$. coli $\mathrm{O} 157: \mathrm{H} 7$ and S. Typhimurium, but more research is needed prior to establishing these techniques for food packaging applications (Sharma et al., 2017). An indirect measure of microbial contamination is provided by freshness indicators. In this case the intelligent device is able to react with a microbial metabolite, giving visual information about the freshness of the product. Smolander et al. (2004) developed a food packaging material consisting of plastic film or paper coated with a very thin silver or copper layer that reacts to the presence of sulfide volatiles produced by fresh meat undergoing spoilage, inducing a color change. Another important group consists of systems that indicate the presence of volatile amines, which are indicators of fish freshness (Kuswandi et al., 2012; Ma et al., 2018; Maynor et al., 2007). Time temperature indicators can also be considered as "freshness indicators", as temperature abuse during distribution and storage of foods largely determines their physical and microbial spoilage (Angiolillo et al., 2017). Zhang et al. (2013) developed a time temperature intelligent system constructed from plasmonic nanocrystals that can be specifically tailored to 
react in a specific temperature range and thus be used to track perishable food products, indicating product quality through sharp-contrast multicolor changes.

\section{BIOSENSORS FOR DETECTION OF COMPOUNDS OR MICROORGANISMS}

Among the techniques for evaluating quality as for what concerns quality control, food safety, and traceability of food products, and specifically of seafood meats and derived products, in recent years the potential use of biosensors stands out. They allow interaction with the chemical compound to be determined with high sensitivity in a fast and economic way.

The biosensors most commonly used or that stand out as interesting for their application are of various kinds, depending on which compound, or even microorganism, they are intended to detect. In many cases they have not yet been implemented and are mere prototypes, but soon we will be surprised by the total implementation of these devices in commerce because their responses are extraordinary in terms of sensitivity, achieving lower detection limits, speed, and cost.

Regardless of the compound to be determined, there are several types of biosensors. In this chapter we will refer to nanosensors. Nanoscale materials have unique properties such as having a greater surface area, increased surface reactivity, electrical conductivity, and magnetic properties (Inbaraj and Chen 2016), and therefore could be used to design detection devices that improve sensitivity and reduce detection time and cost. In addition, nanomaterial is very suitable for functionalizing; it can attach to a wide diversity of biomolecules that give it functionality to detect or recognize biological analytes or bioagents on the surface or nanomaterial. For this purpose it should have suitable charges and structure orientation.

The following describes some types of nanobiosensors developed as potential substitutes for traditional methods to quickly and sensitively analyze certain compounds or microorganisms in 
meat, fish, or seafood and derived products that lead to quality loss. Obviously, the classification is not strict, because there can be overlaps, depending on the systems.

\section{Nucleotide or peptide sequence-based nanoparticle sensors}

This type of sensor has immediate application in the identification of species; therefore its most immediate use may be for detection of meat adulteration, and for identification of microorganisms, focusing on identification of pathogenic organisms in fresh or processed meats or seafood.

Basically it consists in coupling a fragment of oligonucleotide (very characteristic of the species in question) to a nanocrystal, often nanoparticles of gold, which are stabilized in turn by covering them with a solution. When it comes into contact with the sample to be identified, the oligonucleotide fragment, if it is of the selected species, will be recognized and a conformational change will be produced favoring the fluorescence signal of the fluorophore that has at one end the oligonucleotide with which it was labelled previously (Ali et al., 2012).

In this regard, Ali et al. (2012) fabricated a sensor that has been successfully tested to detect adulteration with meat such as beef, mutton, chevon, or chicken in pork meatballs. For this purpose, a 27-nucleotide Alul fragment of pork cytochrome b (cytb) gene was joined to $3 \mathrm{~nm} \varnothing$ citrate-tannate-coated gold nanoparticles. The biosensor was applied to evaluate the amount of pork meat in meatball formulations (in both raw and cooked). Two variations of the sensor were made, as described by Ali et al., 2012, 2014). The differences mainly lie in the detection method, either by fluorescence (Ali et al., 2012) or by colorimetry (Ali et al., 2014). The sensor was sensitive to detect $1 \%$ of pork meat in meatballs (raw and cooked), elaborated from formulations containing pork and beef previously mixed in different proportions. Recently, a group of researchers modified this probe design with thiol groups, giving a stronger bond with gold nanoparticles (Ardhiyana et al., 2017). As in previous works, it was confirmed that the 
biosensor does not react with DNA samples belonging to other species such as beef and chicken, the limit of detection being $16.04 \mathrm{ng} \mathrm{DNA} / \mu \mathrm{l}$.

It has also been applied for the determination of pathogens frequent in meat and fishery products, such as L. monocytogenes (Yang et al., 2017), using a conjugated DNA fragment or a specific peptide on which a protease of the microorganism acts (Alhogail et al., 2016).

\section{Nano-gold particle electrode modified by L-cys and glucose oxidase complex}

Umuhumuza and Sun (2011) manufactured a sensor to measure glucose in pork meat, using the longissimus dorsi muscle, to evaluate the effectiveness of this system in comparison with the traditional measurement determined by HPLC. Glucose is a measure of meat quality and determines its quality/freshness. In broad outline, this sensor consisted of a gold nanoparticle electrode to which L-cys was bound, forming monolayers through the thiol groups, complexing the polyglutamate oxidase enzyme, and leaving free the carboxylic and amino groups that could interact with biological molecules. When the sample of longissimus dorsi pork muscle preserved for 5 days at $4{ }^{\circ} \mathrm{C}$ was passed through the electrode, the values obtained showed a similar evolution and data very close to those obtained by HPLC, and without significant differences in almost all the points analyzed. Therefore, this type of nanosensor stands out as a quick and reliable method, of great interest for implementation in the industry as a method for detecting glucose in meat.

\section{Sulfide nanoparticle amperometric sensor}

The objective of this nanosensor is to use a simple, reliable, fast method to estimate residues of chloramphenicol in meat (beef, pork, chicken), therefore easy to implement, as a new alternative to traditional methods (chromatographic, immunological, etc.), which are timeconsuming and expensive (Kim et al., 2010). An amperometric immunosensor was used to determine chloramphenicol, based on covalent immobilization of anti-chloramphenicol 
acyltransferase antibody on a system of cadmium nanoparticles that act as an electrode, modified with dendrimers of polyamidoamine and gold nanoparticles (Au NP), which are applied to improve the sensitivity of the dendrimer, and a conductive polymer (poly 5, $2^{\prime}: 5^{\prime}, 2^{\prime \prime}$ terthiophene-3'-carboxyl acid (poly-TTCA)) (Kim et al., 2010). The system thus assembled contained a high quantity of amine groups available in the poly-TTCA/Au NP/dendrimer layer and free carboxylic groups in cadmium sulfide nanoparticles. The immunosensor for chloramphenicol exhibited a low detection limit, $45 \pm 5.0 \mathrm{pg} / \mathrm{mL}$, an analogous result to that obtained by HPLC, and to those obtained by chemiluminescence immunosensor ( $100 \mathrm{pg} / \mathrm{mL}-10$ $\mathrm{ng} / \mathrm{mL}$ ) (Park and Kim, 2006). These authors also studied whether the immunosensor detected other antibiotics (ampicillin, streptomycin, tetracycline, neomycin, in 0.1\% BSA (bovine serum albumin)), but it did not respond to significant concentrations.

\section{Quantum dot sensors}

Quantum dot sensors consist of a crystalline cluster with a nano-sized quantum-confined semiconductor. They have unique properties, such as their small size, energy-dependent emission, and excellent optical characteristics. They also present easy surface functionalization, which makes them very suitable for the detection of analyte through labeling or bioimaging due to changes in light emission properties (Rowland et al., 2016; Pérez-López \& Merkoci, 2011). They can be synthesized from various materials: cadmium sulfide, cadmium selenide, cadmium telluride, etc.

In the last few years there have been many studies related to this type of nanobiosensor, with various adaptations in their functionalization, mostly intended to be used for the determination of residues of toxic substances in meat and fish products and illegal use of substances such as clenbuterol and ractopamine in animal feed.

Zhang et al. (2016) described a sensor that consisted of zinc sulfide quantum dots and polyaniline that was deposited on a gold nanoparticle electrode together with antibody against 
clenbuterol that resulted in an amperometric immunosensor for this substance. The biosensor has a detection limit of $5.5 \mathrm{pg} / \mathrm{mL}$. It was also verified that ractopamine and salbutamol (related species) did not interfere. A somewhat less sensitive nanobiosensor (10-12 g/mL) was also developed previously by Tran et al. (2015) to determine clenbuterol. In this sensor the fluorescence resonance energy transfer (FRET) effect was used to fabricate a system based on cadmium telluride quantum dots. Attempts have also been made to detect residual ractopamine in meat by means of optical biosensors. A system to detect ractopamine was developed for this purpose, supported by the fact that fluorescent polymer dots (positive readout) in close proximity to gold nanoparticles produce a strong fluorescence quenching (Shi et al., 2015).

This type of compounds with hormonal effect in animals such as clenbuterol, ractopamine, etc, are not the only ones for the determination of which this type of nanobiosensor has been applied. Among other examples can be mentioned, Hu et al. (2017) developed one for the determination of sulfaquinoxaline, which is a type of sulfonamide, an antibiotic whose presence in foods of animal origin is not allowed or is allowed only in limited concentrations, depending on the country. The detection limit for this antibiotic was $1 \mathrm{ng} / \mathrm{mL}$ in standard solution and 8 $\mathrm{mg} / \mathrm{kg}$ in the samples tested; the products tested being chicken and shrimp meat.

\section{Carbon-based nanomaterials}

Their use in biosensors is highly appreciated because of their biocompatibility and high electrical conductivity using photophysical and fluorescent properties, and their very diverse functionalization. They present many different properties, depending on their structure, metal semiconductor, and polymeric nanomaterial, owing to their good surface effects, small size, and quantum tunnel effect (Afreen et al., 2015).

As in the previous case of quantum dots, most of the studies found in the literature for determinations in meat products focus mainly on the determination of trace or non-permitted compounds such as clenbuterol. These nanosensor systems present various functionalization 
strategies; for example, Liu et al. (2017) have been developing a fluorescence resonance energy transfer (FRET) system consisting of carbon dots and gold nanoparticles for recovered fluorescence detection of clenbuterol in pork meat. The limit of detection for clenbuterol was 3 $\mathrm{nM}$, and the results obtained with the sensor were similar to those obtained by HPLC analysis (for example, $20.9 \mathrm{nM}$ versus $18.75 \mathrm{nM}$ ), demonstrating that it could be used as a fast, reliable, cost-effective method for determination of clenbuterol traces in pork meat samples.

\section{Xanthine oxidase immobilization systems}

Special attention should, perhaps, be paid to xanthine oxidase immobilization systems with nanobiosensors; at any rate, much development work has been done on them in recent years. Devi et al. (2012) described an amperometric xanthine biosensor based on immobilization of xanthine oxidase on a zinc oxide/chitosan/multiwalled carbon nanotube/polyaniline composite film. Subsequently, Dalkiran et al. (2014) described an amperometric sensor based on immobilization of xanthine oxidase on a wall of chitosan/carbon nanotubes modified with glassy carbon electrode. Devi et al. (2013) described another amperometric biosensor, a modification of the previous one, for the determination of xanthine, based on covalent immobilization of xanthine oxidase in a chitosan/Fe-NPs-Au film. They studied both the reuse of the electrode enzyme and its stability, and they tested fish samples in chilled storage $\left(4^{\circ} \mathrm{C}\right)$ during their shelf life. The authors observed that with this modification they improved the sensitivity with respect to that reported in Devi et al. (2013), and they attributed the good stability, repeatability, and reproducibility to the covalent immobilization. However, the electrode showed a $25 \%$ loss after 100 uses. Later, an immobilization of xanthine oxidase covalently bound to a graphite electrode coated with the copolymer poly GMA-co VFc (glycidyl methacrylate and vinylferrocene) was developed by Dervisevic et al. (2015). The results of xanthine in fish samples stored at $4{ }^{\circ} \mathrm{C}$ were very accurate. Albeda et al. (2017) manufactured an amperometric nanosensor of hypoxanthine, based on an electrode consisting of a nanocomposite of graphene/titanium dioxide in which 
xanthine oxidase was immobilized. for evaluation in pork meat. Development of a glassy carbon electrode coated with chitosan-polypyrrole-gold nanoparticles and with subsequent immobilization of xanthine oxidase was carried out by Dervisevic et al. (2017). Various samples of fish, beef, and chicken were tested in the electrode with precise and repetitive results. In general, the lowest detection limits oscillate around $9.0 \mu \mathrm{M}$ in the sensors described, although some of them show a somewhat higher limit, and the analysis ranges are very variable.

\section{HPLC electrochemical analyses with nanoparticulate electrodes}

An electrochemical detection method coupled to HPLC to evaluate possible adulteration of meat with meat of another species has been developed by Hung et al. (2011), based on previous studies. With this method they aim to differentiate ostrich meat from other meats such as chicken, beef, and pork. The method basically consists of an HPLC coupled to an electrochemical analyzer with 3 electrodes (a silkscreen electrode plated with copper nanoparticles, an $\mathrm{Ag} / \mathrm{AgCl}$ reference electrode, and an auxiliary platinum electrode). Lysine, carnosine, anserine, and glutamine are detected in the chromatographic profiles. The results indicate that ostrich meat is easily distinguishable from the others owing to the absence of carnosine, while beef and pork manifest absence of anserine, so they can be differentiated from the poultry myosystem (ostrich and chicken). Therefore this method could be suitable for incorporation as a routine method to differentiate these myosystems in processed meats.

\section{Layered double hydroxides (LDHs)}

Layered double hydroxides are structures formed by layers of positively charged metal hydroxides and they are stabilized with interlaminar anions and water. The interlaminar distance can be at the nanoscale. The negative charge on the surface of the layers, owing to the hydroxyl groups, and the interchangeable anions make them ideal systems for preparing functionalization products, hybridizing molecules, or acting as biosensors, allowing a wide range of applications to be carried out at the nanometer scale, such as catalysts, intercalation products, etc. Basically, 
they consist of a magnesium hydroxide structure where magnesium is centrally arranged in the layers and hydroxides on the surface. LDHs are the result of substitution of a fraction of (divalent) magnesium cations by a trivalent cation, which generates a residue with a positive charge in the layers. Intercalation is a term frequently used to refer to the introduction of a great variety of organic species and ions between the layers. They can also be intercalated in a single step, called coprecipitation (Martínez \& Carbajal, 2012).

In addition, a measurable signal must be shown, for example chemiluminescence, which has a fast response, high sensitivity, and cheap and easy implementation, so its application in food is highly useful.

An application of this system has been used for determination of biogenic amines in fish during deterioration, as well as in pork. The results were carried out with simplicity, selectivity, and sensitivity (Wang et al., 2014.) In general, the method used was coprecipitation for $\mathrm{Mg}-\mathrm{Al}-\mathrm{CO}_{3}$, with $\mathrm{Mg} / \mathrm{Al}$ and exfoliation in formamide solution, favoring delamination through hydrogen bonds, which leads to strong luminescence of the exfoliated nano-LDH. This photoluminescence decreases in the presence of biogenic amines, owing to displacement of the $\mathrm{O}-\mathrm{H} \ldots \mathrm{O}$ bond by the $\mathrm{O}-\mathrm{H} \ldots \mathrm{N}$ bond (Wang et al., 2014).

\section{Nanoporous colorimetric sensor for detection of trimethylamine}

Various authors coincide in using this type of nanosensor for the determination of volatile compounds such as amines. The colorimetric sensor array must contain a functional group to interact strongly with the analytes, and this functional group should be firmly coupled to a powerful chromophore (Xiao-Wei et al., 2014). Xiao-Wei et al. (2016) created a nanoporous structure as a gas sensor sensitive to the detection of trimethylamine (TMA) in Yao meat, which is a typical salted Chinese meat. TMA is one of the volatile organic compounds that allow evaluation of the freshness of meat, fish, and seafood owing to its production and release in animal tissues as a result of microbial deterioration of these myosystems. The determination in 
the sensor is based on the faster diffusion of the analyte gases on the porous sensor surface. To do this, nanoporous colorimetric matrices of chemosensitive dyes were created, for which the dyes were printed on a plate of nanoporous $\mathrm{TiO}_{2}$ film. The TMA was collected by means of a conductive gas that was deposited on the porous film, which was dyed in a known range of colors, depending on the concentration of TMA. This system was very sensitive to low concentrations of TMA and was repeatable.

Morsy et al. (2016) applied another colorimetric sensor array to monitor fish spoilage compounds, including various amines. The chemosensor contained an array of 16 chemosensitive compounds in conditions of response to standard volatile compounds that are produced by fresh fish or as a result of fish spoilage during storage. This sensor detects changes in fish muscle $\mathrm{pH}$ and in the thiobarbituric acid with great reliability, but not detect amines produced. During storage, it shows a linear correlation with the increase in microorganisms, responding to differences due to storage conditions (room and $4{ }^{\circ} \mathrm{C}$ ). With a similar system, Xiao-Wei et al. (2014) created a colorimetric gas sensor matrix based on four highly sensitive natural pigments for the determination of freshness (microbial growth and biogenic amines) in pork meat stored at $4{ }^{\circ} \mathrm{C}$. The results established good correlations with the data obtained by conventional analyses. Therefore, these authors consider that it is a viable method for evaluating total microbial growth and the biogenic amine index.

\section{Aptasensors}

Aptasensors are basically nanosensors in which aptamers are immobilized. Aptamers are single-stranded oligonucleotides with sizes between 70 and 100 nucleotides, with high affinity to various types of analytes or specific molecules, and capable of specifically recognizing them by means of a 3D structure. The aptamer is usually obtained by selection in the SELEX method (Systematic Evolution of Ligands by Exponential Enrichment), with high affinity and specificity for a specific analyte. Consequently, aptamers are used as probes in sensors; they have high 
stability at room temperature, it is easy to mark the nucleotide sequence with other molecules (that is, chemically label the aptamer for later identification), and they can be synthesized chemically and at low cost.

The aptasensor preparation is determined by the aptamer immobilization method, because the sensitivity and selectivity of the assay will be affected by the orientation and accessibility of the receiver. To facilitate interaction of the immobilized aptamer with the ligand in solution, it is common to add a spacer by means of a suitable functional group.

Prototypes of aptasensors have been created for the detection of chemical compounds such as antibiotics or anabolic steroids that could remain in meat, and for the detection of microorganisms. The number of aptasensors for an analyte determination can vary greatly owing to many factors, such as type of sensor, labelling etc.

In the last few years several aptasensors have been developed for the determination of clenbuterol. Duan et al. (2017) tested a fluorescent method based on a specific aptamer for clenbuterol by adsorption to graphene oxide, which was used to confirm its utility as a recognition receptor with high affinity and specificity for the detection of clenbuterol in pig samples. The measurement showed good sensitivity in the range of 0.10 to $50 \mathrm{ng} / \mathrm{mL}$ and a detection limit of $0.07 \mathrm{ng} / \mathrm{mL}$. In samples of pork meat the results fitted this range perfectly, which means the aptasensor could be applied to this type of product.

A colorimetric aptasensor was created for sensitive and selective detection of chloramphenicol based on gold nanoparticles (Au NPs) labelled with Power Vision (PV) and magnetic separation (Gao et al., 2015). The chloramphenicol aptamer was immobilized on $\mathrm{Fe}_{3} \mathrm{O}_{4} @ A u$ magnetic nanoparticles as a capture probe (AuMNPs-Apt) to concentrate target chloramphenicol. The power vision system consists of peroxidase enzyme and conjugated antibody. The color evolution produced can be measured by ultraviolet-visible (UV-vis) spectroscopy. The responses 
to chloramphenicol concentration were in a range of $0.05-200 \mathrm{ng} / \mathrm{mL}$, with a detection limit of $0.02 \mathrm{ng} / \mathrm{mL}$. This test was successfully applied to analyze chloramphenicol in fish and pork muscle pieces, and the results were according with those of the conventional enzyme-linked immunosorbent test (ELISA).

More recently, however, Ouyang et al. opted for another kind of aptasensor for detection of traces of tetracycline in pork and milk (Ouyang et al., 2017). The design was based on those mentioned before, using nanoparticles of $\mathrm{Fe}_{3} \mathrm{O}_{4}$ functionalized with amine and modifying the surface with lanthanide doping. The nanoparticles were conjugated with the aptamer. When the sample of pork with tetracycline (or any kind of food containing tetracycline) was added, the aptamer preferentially bonded with tetracycline to cause the dissociation of some cDNA and the liberation of some cDNA-nanosensor, producing a decrease in the fluorescent signal. The results indicate that the aptasensor is very specific for this antibiotic, as in the presence of others it only recognizes tetracycline. The detection in samples of adulterated meat and milk showed great repeatability of the method and an amplitude range in the measurement $(0.01-100 \mathrm{ng} / \mathrm{mL}$, with a limit of detection of $0.0062 \mathrm{ng} / \mathrm{mL}$ ) that makes it ideal for determination in real samples to ensure their safety.

As mentioned earlier, aptasensors can also be used for the identification of microorganisms, and some of them will be mentioned as case studies. The surface-enhanced properties of Raman scattering (SERS) nanomaterials have also been used to develop sensors for the detection of microorganisms, especially pathogens. The pathogenicity of certain bacteria such as Salmonella Typhimurium, Listeria monocytogenes, etc. is well known, and also the ease with which they contaminate certain foods, especially meat products. For this reason, development of this type of sensor for these types of bacteria has begun. As nanoprobes Ma et al. (2018) used SERS (surface-enhanced Raman scattering) spiny gold nanoparticles immobilized with molecules with Raman signal and a thiolated aptamer for S. thyfimurium. The aptasensor responds specifically 
against $S$. thyfimurium owing to the high affinity and specificity shown by the aptamer, with a detection limit of $4 \mathrm{cfu} / \mathrm{mL}$ in inoculated pork meat.

Shahdordizadeh et al. (2017) describe 3 kinds of aptasensors for the detection of Staphylococcus aureus: colorimetric, electrochemical, and fluorescent. While the colorimetric type is the simplest and the result is seen with the naked eye, the electrochemical type shows the highest sensitivity and a low limit of detection $(1 \mathrm{cfu} / \mathrm{mL})$, attributed to the sensor, based on reduced graphene, which has very good conductivity. Finally, the fluorescent aptasensor presented a good signal for S. aureus (8 $\mathrm{cfu} / \mathrm{mL})$ and for the toxin that it produces $(6 \mathrm{ng} / \mathrm{mL})$.

\section{CONCLUDING REMARKS}

The enrichment of meat and fishery products with natural active compounds added freely or encapsulated in diverse nanoestructures, such as chitosan nanoparticles, liposomes or emulsions, is a field with great prospects for industrial and market development. These nanosystems not only may favour the controlled release of antioxidants or antimicrobial agents to enhance the quality and durability of the product, but also they have great potential in the design of versatile functional foods.

Nanotechnology can be also applied to the design of food packaging materials with improved properties, such a better physico-chemical performance or an active/intelligent activity, thus improving fish and meat quality during storage.

Most of the nanobiosensors that are being designed today are very diverse and are based on their electrical, magnetic, luminescent, etc properties of nanomaterials. In addition, they are very suitable to be functionalized, that is, capable of joining a great variety of biomolecules that enable it to detect biological analytes on the surface of nanomaterials, confering specifyty them. Among the most widely studied compounds include anabolic in meat as clenbuterol, biogenic 
amines in meat, poultry and fish, as well as detection of microorganisms, in special, certain pathogens, what gives an idea of the versatility in terms of their applications

\section{ACKNOWLEDGEMENTS}

The authors wish to thank the Spanish Ministry of Economy and Competitiveness for financial support through AGL2014-52825-R and AGL2017-84161-C2-1 projects, both co-funded with EURegional Development Fund.

\section{REFERENCES}

Afreen, S., Muthoosamy, K., Manickam, S. and Hashim, U. 2015. Functionalized fullerene (C60) as a potential nanomediator in the fabrication of highly sensitive biosensors, Biosens. Bioelectron. 63, 354-364.

Akbar, A., \& Anal, A. K. 2014. Zinc oxide nanoparticles loaded active packaging, a challenge study against Salmonella typhimurium and Staphylococcus aureus in ready-to-eat poultry meat. Food Control, 38 (1), 88-95.

Akbarzadeh, A., Rezaei-Sadabady, R., Davaran, S., Joo, S.W., Zarghami, N., Hanifehpour, Y., Samiei, M., Kouhi, M., Nejati-Koshki, K. 2013. Liposome: Classification, preparation, and applications. Nanoscale Research Letters, 8 (1), 1-8.

Al-Adham, I.S.I., Khalil, E., Al-Hmoud, N.D., Kierans, M., \& Collier, P.J. 2000. Microemulsions are membrane-active, antimicrobial, self-preserving systems. Journal of Applied Microbiology 89:32-39.

Albelda, J., Uzunoglu, A., Santos, G.N.C. and Stanciu, L.A. 2017. Graphene-titanium dioxide nanocomposite based hypoxanthine sensor for assessment of meat freshness. Biosensors and Bioelectronics 89, 518-524.

Alboofetileh, M., Rezaei, M., Hosseini, H., \& Abdollahi, M. 2016. Efficacy of activated alginatebased nanocomposite films to control Listeria monocytogenes and spoilage flora in rainbow trout slice. Journal of Food Science and Technology, 53 (1), 521-530. 
Alemán, A., Mastrogiacomo, I., López-Caballero, M.E., Ferrari, B., Montero, M.P., GómezGuillén, M.C. 2016. A Novel Functional Wrapping Design by Complexation of $\varepsilon$-Polylysine with Liposomes Entrapping Bioactive Peptides. Food and Bioprocess Technology, 9 (7), 1113-1124.

Alhogail, S., Suaifan, G.A.R.Y., Zourob, M. 2016. Rapid colorimetric sensing platform for the detection of Listeria monocytogenes foodborne pathogen. Biosensors and Bioelectronics 86, 1061-1066.

Ali, M. E., Hashim, U., Mustafa, S., Che Man, Y.B. and Islam, Kh. N. 2012. Gold Nanoparticle Sensor for the Visual Detection of Pork Adulteration in Meatball Formulation. Journal Nanomater, 103607, 1-7. doi:10.1155/2012/103607

Ali, M.E., Hashim, U., Mustafa, S., Che Man, Y.B., Adam, T. and Humayun, Q. 2014. Nanobiosensor for the detection and quantification of pork adulteration in meatball formulation. Journal of Experimental Nanoscience, 9 (2), 152-160

Alishahi, A., A. Mirvaghefi, M. R. Tehrani, H. Farahmand, S. Koshio, F. A. Dorkoosh, and Maher Z. Elsabee. 2011. Chitosan nanoparticle to carry vitamin C through the gastrointestinal tract and induce the non-specific immunity system of rainbow trout (Oncorhynchus mykiss). Carbohydrate Polymers 86 (1):142-146.

Angiolillo, L., Spinelli, S., Conte, A., \& Alessandro Del Nobile, M. 2017. Recent Advances in Food Packaging with a Focus on Nanotechnology. Recent Patents on Engineering, 11(3), 174-187. Ardhiyana, R., Haditjaroko, L., Mulijani, S., Wicaksono, R.A. and Ranasasmita, R. 2017. DNAbased gold nanoprobe biosensor to detect pork contaminant. Rasayan J.Chem. 10 (3), 1037 $-1042$

Arora, A., \& Padua, G. W. 2010. Review: Nanocomposites in food packaging. Journal of Food Science, 75 (1), R43-R49.

Azlin-Hasim, S., Cruz-Romero, M. C., Morris, M. A., Padmanabhan, S. C., Cummins, E., \& Kerry, J. P. 2016. The Potential Application of Antimicrobial Silver Polyvinyl Chloride Nanocomposite 
Films to Extend the Shelf-Life of Chicken Breast Fillets. Food and Bioprocess Technology, 9 (10), 1661-1673.

Bai, J.J., Lee, J.G., Lee, S.Y., Kim, S., Choi, M.J, Cho, Y. 2017. Changes in Quality Characteristics of Pork Patties Containing Antioxidative Fish Skin Peptide or Fish Skin Peptide loaded Nanoliposomes during Refrigerated Storage. Korean Journal of Food Science and Animal Resources, 37(5), 752-763.

Boualem, K., Subirade, M., Desjardin, Y., Saucier, L. 2013. Protection and controlled release of nisin in meat during cooking using liposome encapsulation. CMSA News, April 2013, 19-23.

Bouwmeester, H., Dekkers, S., Noordam, M. Y., Hagens, W. I., Bulder, A. S., de Heer, C., ten Voorde, S. E. C. G., Wijnhoven, S. W. P., Marvin, H. J. P., \& Sips, A. J. A. M. 2009. Review of health safety aspects of nanotechnologies in food production. Regulatory Toxicology and Pharmacology, 53(1), 52-62.

Busolo, M. A., \& Lagaron, J. M. 2015. Antioxidant polyethylene films based on a resveratrol containing Clay of Interest in Food Packaging Applications. Food Packaging and Shelf Life, 6, $30-41$.

Carbone, M., Donia, D.T., Sabbatella, G., Antiochia, R. 2016. Silver nanoparticles in polymeric matrices for fresh food packaging. Journal of King Saud University - Science, 28 (4), pp. 273279.

Cerisuelo, J. P., Alonso, J., Aucejo, S., Gavara, R., \& Hernández-Muñoz, P. 2012. Modifications induced by the addition of a nanoclay in the functional and active properties of an $\mathrm{EVOH}$ film containing carvacrol for food packaging. Journal of Membrane Science, 423-424, 247-256.

Cerisuelo, J. P., Bermudez, J. M., Aucejo, S., Catala, R., Gavara, R., \& Hernandez-Munoz, P. 2013. Describing and modeling the release of an antimicrobial agent from an active PP/EVOH/PP package for salmon. Journal of Food Engineering, 116(2), 352-361.

Chantarasataporn, Patomporn, Preenapha Tepkasikul, Yutthana Kingcha, Rangrong Yoksan, Rath Pichyangkura, Wonnop Visessanguan, and Suwabun Chirachanchai. 2014. Water-based 
oligochitosan and nanowhisker chitosan as potential food preservatives for shelf-life extension of minced pork. Food Chemistry 159:463-470.

Chen, C., Han, D., Cai, C., Tang, X. 2010. An overview of liposome lyophilization and its future potential. Journal of Controlled Release, 142, 299-311.

Chouljenko, A., A. Chotiko, M. J. M. Solval, K. M. Solval, and S. Sathivel. 2017a. Chitosan Nanoparticle Penetration into Shrimp Muscle and its Effects on the Microbial Quality. Food and Bioprocess Technology 10 (1):186-198.

Chouljenko, A., A. Chotiko, F. Bonilla, M. Moncada, V. Reyes, and S. Sathivel. 2017b. Effects of vacuum tumbling with chitosan nanoparticles on the quality characteristics of cryogenically frozen shrimp. LWT - Food Science and Technology 75:114-123.

Cui, H., Yuan, L., Lin, L. 2017a. Novel chitosan film embedded with liposome-encapsulated phage for biocontrol of Escherichia coli 0157:H7 in beef. Carbohydrate Polymers, 177, 156-164.

Cui, H., Yuan, L., Ma, C., Li, C., Lin, L. 2017b. Effect of nanoliposome-encapsulated thyme oil on growth of Salmonella enteritidis in chicken. Journal of Food Processing and Preservation, https://doi.org/10.1111/jfpp.13299

da Silva Malheiros, P., Daroit, D.J., Brandelli, A. 2010. Food applications of liposomeencapsulated antimicrobial peptides. Trends in Food Science and Technology, 21 (6), 284-292.

Dalkian, B., Kaçar, C., Erden, P.E. and Kiliç, E. 2014. Amperometric xanthine biosensors based onchitosan-Co304-multiwall carbon nano tube modified glassy carbon electrode. Sensors and Actuators B 200, 83-91.

Degnan, A. J., Buyong, N., Luchansky, J. B. 1993. Antilisterial activity of pediocin AcH in model food systems in the presence of an emulsifier or encapsulated within liposomes. International Journal of Food Microbiology, 18, 127-138.

Degnan, A. J., Luchansky, J. B. 1992. Influence of beef tallow and muscle on the antilisterial activity of pediocin $\mathrm{AcH}$ and liposome-encapsulated pediocin AcH. Journal of Food Protection, $55,552-554$. 
Dervisevic, M., Custiuc, E., Çevik, E., Şenel, M. 2015. Construction of novel xanthine biosensor by using polymeric mediator/MWCNT nanocomposite layer for fish freshness detection. Food Chemistry, 181, 277-283.

Dervisevic, M., Dervisevic, E., Senel, M., Cevik, E. and Abasiyanik, F.M. 2017. Novel Amperometric Xanthine Biosensors Based on REGO-NP ( $\mathrm{Pt}, \mathrm{Pd}$, and $\mathrm{Au}$ ) Bionanocomposite Film. Food Anal. Methods, 10, 1252-1263.

Devi, R., Yadav, S. and Pundir, C.S. 2012. Amperometric determination of xanthine in fish meat by zinc oxide nanoparticle/ chitosan/ multiwalled carbon nanotube/polyaniline composite film bound xanthine oxidase. Analyst, 137, 754.

Devi, R., Yadav, S., Nehra, R., Yadav, S. and Pundir, C.S. 2013. Electrochemical biosensor based on gold coated iron nanoparticles/ chitosan composite bound xanthine oxidase for detection of xanthine in fish meat. Journal of Food Engineering 115, 207-214.

Donsì, F., Annunziata, M., Sessa, M., and Ferrari, G. 2011. Nanoencapsulation of essential oils to enhance their antimicrobial activity in foods. LWT - Food Science and Technology 44: 190814.

Duan, N., Gong, W., Wu, S. and Wang, Z. 2017. Selection and Application of ssDNA Aptamers against Clenbuterol Hydrochloride Based on ssDNA Library Immobilized SELEX. J. Agric. Food Chem. 65, 1771-1777.

Echeverría, I., López-Caballero, M.E., Gómez-Guillén, M.C., Mauri, A.N., Montero, M.P. 2016. Structure, functionality 1 and active release of nanoclay- soy proteins films affected by clove essential oil. Food and Bioprocess Technology 9 (11): 1937-1950.

Echeverría, I., López-Caballero, M.E., Gómez-Guillén, M.C., Mauri, A.N. and Montero, M.P. 2018. Active composite films based on soy proteins montmorillonite clove essential oil for the preservation of refrigerated Bluefin tuna (Thunnus thynnus) fillets. International Journal of Food Microbiology, 266 (2), 142-149. 
[EFSA] European Food Safety Authority, 2011. Guidance on the risk assessment of the vapplication of nanoscience and nanotechnology in the food and feed chain. EFSA J. 9 (5), 2140.

Espitia, P. J. P., Otoni, C. G., \& Soares, N. F. F. 2016. Zinc Oxide Nanoparticles for Food Packaging Applications. In Antimicrobial Food Packaging, (pp. 425-431).

Fernández, A., Picouet, P., \& Lloret, E. 2010. Reduction of the spoilage-related microflora in absorbent pads by silver nanotechnology during modified atmosphere packaging of beef meat. Journal of Food Protection, 73(12), 2263-2269.

Gan, Q., Wang, T., Cochrane, C. and McCarron. P. 2005. Modulation of surface charge, particle size and morphological properties of chitosan-TPP nanoparticles intended for gene delivery. Colloids and Surfaces B: Biointerfaces 44 (2):65-73.

Gao, H., Gan, N., Pan, D., Chen, Y., Li, T., Cao, Y. and Fua, T. 2015. A sensitive colorimetric aptasensor for chloramphenicol detection in fish and pork based on the amplification of a nano-peroxidasepolymer. Anal. Methods, 7, 6528-6536.

Ghaani, M., Cozzolino, C. A., Castelli, G., \& Farris, S. 2016. An overview of the intelligent packaging technologies in the food sector. Trends in Food Science and Technology, 51, $1-11$.

Ghaderi-Ghahfarokhi, M., M. Barzegar, M. A. Sahari, H. Ahmadi Gavlighi, and F. Gardini. 2017. Chitosan-cinnamon essential oil nano-formulation: Application as a novel additive for controlled release and shelf life extension of beef patties. International Journal of Biological Macromolecules 102:19-28.

Ghaderi-Ghahfarokhi, M.,, Barzegar,M., Sahari, M.A. and Azizi, M.H. 2016. Nanoencapsulation Approach to Improve Antimicrobial and Antioxidant Activity of Thyme Essential Oil in Beef Burgers During Refrigerated Storage. Food and Bioprocess Technology 9 (7):1187-1201. 
Gharibzahedia, S. M. T., and Mohammadnabiba, S. 2017. Effect of novel bioactive edible coatings based on jujube gum and nettle oil-loaded nanoemulsions on the shelf-life of Beluga sturgeon fillets. International Journal of Biological Macromolecules 95: 769-777.

Gokularaman, S., Stalin Cruz, A., Pragalyaashree, M. M., \& Nishadh, A. 2017. Nanotechnology approach in food packaging - Review. Journal of Pharmaceutical Sciences and Research, 9(10), 1743-1749.

Gómez-Estaca, J., Gavara, R., Catalá, R., \& Hernández-Muñoz, P. 2016. The Potential of Proteins for Producing Food Packaging Materials: A Review. Packaging Technology and Science, 29(4-5): 203-224.

Gómez-Estaca, J., López-de-Dicastillo, C., Hernández-Muñoz, P., Catalá, R., \& Gavara, R. 2014. Advances in antioxidant active food packaging. Trends in Food Science and Technology, 35(1), 42-51.

Gültekin-Özgüven, M., Karadağ, A., Duman, S., Özkal, B. \&Özçelik, B. 2016. Fortification of dark chocolate with spray dried black mulberry (Morus nigra) waste extract encapsulated in chitosan-coated liposomes and bioaccessability studies. Food Chemistry, 201, 205-212.

Hashemi. S.R., D. Davoodi, B. Dastar. 2017. Effect of clinoptilolite coated with silver nanoparticles on meat quality attributes of broiler chickens during frozen storage. Iranian Journal of Applied Animal Science 7(2): 321-328.

Henna Lu, F.S., Nielsen, N.S., Timm-Heinrich, M., Jacobsen, C. 2011. Oxidative stability of marine phospholipids in the liposomal form and their applications. Lipids, 46 (1), 3-23.

Hu, G., Sheng, W., Li, J., Zhang, Y., Wang, J. and Wang S. 2017. Fluorescent quenching immune chromatographic strips with quantum dots and upconversion nanoparticles as fluorescent donors for visual detection of sulfaquinoxaline in foods of animal origin. Analytica Chimica Acta 982, 185-192. 
Hung, C., Ho, H., Chang, C., Lee, M., Franje, C., Kuo, S., Lee, R. and Chou, C. 2011. Electrochemical profiling using copper nanoparticle-plated electrode for identification of ostrich meat and evaluation of meat grades. Food Chemistry 126, 1417-1423.

Inbaraj, S., and Chen, B.H. 2016. Nanomaterial-based sensors for detection of foodborne bacterial pathogens and toxins as well as pork adulteration in meat products. Journal of Food and Drug Analysis, 24 (1), 15-28

Javadian, S.R., Shahosseini, S.R., Ariaii, P. 2017. The Effects of Liposomal Encapsulated Thyme Extract on the Quality of Fish Mince and Escherichia coli 0157:H7 Inhibition During Refrigerated Storage. Journal of Aquatic Food Product Technology, 26 (1), 115-123.

Joe, M. M., Chauhan, P. S., Bradeeba, K., Shagol, C., Sivakumaar, P. K., and Sa, T. 2012. Influence of sunflower oil based nanoemulsion (AUSN-4) on the shelf life and quality of Indo-Pacific king mackerel (Scomberomorus guttatus) steaks stored at $20^{\circ} \mathrm{C}$. Food Control 23:564-70.

Khalaf, H.H.; Sharoba, A.M.; El-Tanahi, H.H. and Morsy, M.K. 2013. Stability of antimicrobial activity of pullulan edible films incorporated with nanoparticles and essential oils and their impact on turkey deli meat quality. Journal of Food and Dairy Science, Mansoura Univ. 4 (11): $557-573$.

Kim, D., Rahman, Md.A., Do, M.H., Ban, C. and Shim, Y. 2010. An amperometric chloramphenicol immunosensor based on cadmium sulphide nanoparticles modified-dendrimer bonded conducting polymer. Biosensors and Bioelectronics 25, 1781-1788.

Kim, J.-M., Kim, Y.-J., Jeong, J., Kim, C.-J. 2006. Meat tenderizing effect of injecting encapsulated $\mathrm{Ca} 2+$ in liposome into rabbit before slaughter. Bioscience, Biotechnology and Biochemistry, $70(10), 2381-2386$

Kuswandi, B., Jayus, Restyana, A., Abdullah, A., Heng, L. Y., \& Ahmad, M. 2012. A novel colorimetric food package label for fish spoilage based on polyaniline film. Food Control, 25(1), 18-189 
Laye, C., McClements, D.J., Weiss, J. 2008. Formation of biopolymer-coated liposomes by electrostatic deposition of chitosan. Journal of Food Science, 73 (5), N7-N15.

Lee, S. K., Sheridan, M., \& Mills, A. 2005. Novel UV-activated colorímetric oxygen indicator. Chemistry of Materials, 17(10), 2744-2751.

Li, F., Mascheroni, E., \& Piergiovanni, L. 2015. The potential of nanocellulose in the packaging field: A review. Packaging Technology and Science, 28(6), 475-508.

Lin, L., Dai, Y., Cui, H. 2017. Antibacterial poly(ethylene oxide) electrospun nanofibers containing cinnamon essential oil/beta-cyclodextrin proteoliposomes. Carbohydrate Polymers, 178, $131-140$.

Liu, Y., Lu, Q., Hu, X., Wang, H., Li, H., Zhang, Y., Yao, S. 2017. A Nanosensor Based on Carbon Dots for Recovered Fluorescence Detection Clenbuterol in Pork Samples. Journal of Fluorescence, 27 (5), 1847-1853.

Lloret, E., Fernandez, A., Trbojevich, R., Arnau, J., \& Picouet, P. A. 2016. Relevance of nanocomposite packaging on the stability of vacuum-packed dry cured ham. Meat Science, 118, 8-14.

López-Carballo, G., Gómez-Estaca, J., Catalá, R., Hernández-Muñoz, P., \& Gavara, R. 2012. Active antimicrobial food and beverage packaging. In Emerging Food Packaging Technologies: Principles and Practice, (pp. 27-54).

Lopez-Rubio, A., Almenar, E., Hernandez-Munoz, P., Lagaron, J. M., Catala, R., \& Gavara, R. 2004. Overview of active polymer-based packaging technologies for food applications. Food Reviews International, 20(4), 357-387.

Luo, Z., Xu, Y., Ye, Q. 2015a. Effect of nano-SiO2-LDPE packaging on biochemical, sensory, and microbiological quality of Pacific white shrimp Penaeus vannamei during chilled storage. Fish Science, 81:983-993. 
Luo, Z., Qin, Y., Ye, Q. 2015b. Effect of nano-TiO2-LDPE packaging on microbiological and physicochemical quality of Pacific white shrimp during chilled storage. International Journal of Food Science and Technology, 50:1567-1573

Ma, X., Xu, X., Xia, Y. And Wang, Z. 2018. SERS aptasensor for Salmonella typhimurium detection based on spiny gold nanoparticles. Food Control 84, 232-237.

Mahdi, S.S.. R. Vadood, R. Nourdahr. 2012. Study on the Antimicrobial Effect of Nanosilver Tray Packaging of Minced Beef at Refrigerator Temperature. Global Veterinaria 9 (3): 284-289.

Marcous, A., Rasouli, S., \& Ardestani, F. 2017. Low-density Polyethylene Films Loaded by Titanium Dioxide and Zinc Oxide Nanoparticles as a New Active Packaging System against Escherichia coli 0157:H7 in Fresh Calf Minced Meat. Packaging Technology and Science, 30(11), 693-701.

Marín, D., Alemán, A., Sánchez-Faure, A., Montero, P., Gómez-Guillén, M.C. 2018a. Freeze-dried phosphatidylcholine liposomes encapsulating various antioxidant extracts from natural waste as functional ingredients in surimi gels. Food Chemistry, 245, 525-535.

Marín, D., Alemán, A., Montero, P., Gómez-Guillén, M.C. 2018b. Encapsulation of food waste compounds in soy phosphatidylcholine liposomes: Effect of freeze-drying, storage stability and functional aptitude. Journal of Food Engineering, 223, 132-143.

Marín, D., Alemán, A., Montero, P., Gómez-Guillén, M.C. 2018c. Protein aggregation, water binding and thermal gelation of salt-ground hake muscle in the presence of wet and dry soy phosphatidylcholine liposomes. Food Hydrocolloids 82, 466-477.

Martínez, D.R. and Carbajal G.G. 2012 Hidróxidos dobles laminares: arcillas sintéticas con aplicaciones en nanotecnología. Avances en Química, 7(1), 87-99.

Martínez-Abad, A., JM. Lagarón, M.J. Ocio. 2012. Development and Characterization of SilverBased Antimicrobial Ethylene-Vinyl Alcohol Copolymer (EVOH) Films for Food-Packaging Applications. Journal of Agricultural and Food Chemistry 60, 5350-5359. 
Maynor, M. S., Nelson, T. L., O'Sullivan, C., \& Lavigne, J. J. 2007. A food freshness sensor using the multistate response from analyte-Induced aggregation of a cross-reactive poly(thiophene). Organic Letters, 9(17), 3217-3220.

McClements, D. J., and Rao, J. 2011. Food-grade nanoemulsions: Formulation, fabrication, properties, performance, biological fate, and potential toxicity. Critical Reviews in Food Science and Nutrition 51:285-330.

McClements, D.J., 2011. Edible nanoemulsions: fabrication, properties, and functional performance. Soft Matter 7:2297-2316.

McClements, D.J., Decker, E. A., and Weiss, J. 2007. Emulsion-based delivery systems for lipophilic bioactive components. Journal of Food Science 72: 109-124.

McMillin, K. W. (2017). Advancements in meat packaging. Meat Science, 132, 153-162.

Mills, A., \& Hazafy, D. (2009). Nanocrystalline SnO2-based, UVB-activated, colourimetric oxygen indicator. Sensors and Actuators, B: Chemical, 136(2), 344-349.

Mirhosseini, M., Arjmand, V. 2014. Reducing pathogens by using zinc oxide nanoparticles and acetic acid in sheep meat. Journal of Food Protection, 77 (9), pp. 1599-1604.

Mishra, P., Kumar, S., Jerobin, J., Thomas, J., Mukherjee, A., and Chandrasekaran, N. 2014. Study on antimicrobial potential of neem oil nanoemulsion against Pseudomonas aeruginosa infection in Labeo rohita. International Union of Biochemistry and Molecular Biology 61: 61119.

Mohammadi, N., Tukmechi, A. 2015. The effects of iron nanoparticles in combination with Lactobacillus casei on growth parameters and probiotic counts in rainbow trout (Oncorhynchus mykiss) intestine. Journal of Veterinary Research, 70 (1), pp. 47-53.

Mokarizadeh, M., Kafil, H.S., Ghanbarzadeh,m S., Alizadeh, A., Hamishehkar, H. 2017. Improvement of citral antimicrobial activity by incorporation into nanostructured lipid carriers: a potential application in food stuffs as a natural preservative. Research in Pharmaceutical Sciences 12(5): 409-415. 
Monroig, O., Navarro, J.C., Amat, F., Gonzalez, P., Bermejo, A., Hontoria, F. 2006. Enrichment of Artemia nauplii in essential fatty acids with different types of liposomes and their use in the rearing of gilthead sea bream (Sparus aurata) larvae. Aquaculture 251:491-508

Monroig, O., Navarro, J.C., Amat, F., Hontoria, F. 2007. Enrichment of Artemia nauplii in vitamin A, vitamin C and methionine using liposomes. Aquaculture 269, 504-513

Montero, P., Giménez, B., Pérez-Mateos, M., Gómez-Guillén, M.C. 2005. Oxidation stability of muscle with quercetin and rosemary during thermal and high-pressure gelation. Food Chemistry, 93, 17-23.

Montero, P., Mosquera, M., Marín-Peñalver, D., Alemán, A., Martínez-Álvarez, Ó., GómezGuillén, M.C. 2019. Changes in structural integrity of sodium caseinate films by the addition of nanoliposomes encapsulating an active shrimp peptide fraction. Journal of Food Engineering 244, 47-54

Mor, Y., Shoemaker, C.F., Rosenberg, M. 1999. Compressive Properties of Whey Protein Composite Gels Containing Fractionated Milkfat. Journal of Food Science, 64, 1078-1083.

Moraes-Lovison, M, Marostegan, L., Peres, M. S., Menezes, I. F., Ghiraldi, M., Rodrigues, R., Fernandes, A. M., and Pinho, S. C. 2017. Nanoemulsions encapsulating oregano essential oil: Production, stability, antibacterial activity and incorporation in chicken pate. LWT - Food Science and Technology 77: 233-40.

Morsy, M.K., Khalaf, H.H., Sharoba, A.M., El-Tanahi, H.H., Cutter, C.N. 2014. Incorporation of Essential Oils and Nanoparticles in Pullulan Films to Control Foodborne Pathogens on Meat and Poultry Products. Journal of Food Science, 79 (4), pp. M675-M684.

Morsy, M.K., Zor, K., Kostesha, N., Alstrøm , T.S., Heiskanen, A., El-Tanahi, H., Sharoba, H., Papkovsky, D., Larsen, J., Khalaf, H., Jakobsen, M.H. and Emnéus, J. 2016. Development and validation of a colorimetric sensor array for fish spoilage monitoring. Food Control 60, 346352 
Morsy; M.K., Mekawib, E., Elsabagh, R. 2018. Impact of pomegranate peel nanoparticles on quality attributes of meatballs during refrigerated storage. LWT - Food Science and Technology 89:489-495.

Mosquera, M., Giménez, B., Montero, P., Gómez-Guillén, M.C. 2016. Incorporation of liposomes containing squid tunic ACE-inhibitory peptides into fish gelatin. Journal of the Science of Food and Agriculture, 96 (3), 769-776.

Mozafari, M.R., Johnson, C., Hatziantoniou, S., Demetzos, C. 2008. Nanoliposomes and their applications in food nanotechnology. Journal of Liposome Research 18: 309-327.

Mufamadi, M. S., Pillay, V., Choonara, Y.E., Du Toit, L.C., Modi, G., Naidoo, D., Ndesendo, V.M., 2011. A review on composite liposomal technologies for specialized drug delivery. Journal of Drug Delivery, 939851.

Natale, D., Gibis, M., Rodriguez-Estrada, M.T., Weiss, J. 2014. Inhibitory effect of liposomal solutions of grape seed extract on the formation of heterocyclic aromatic amines. Journal of Agricultural and Food Chemistry, 62 (1), 279-287.

Navas-Carretero, S., Pérez-Granados, A.M., Sarriá, B., Vaquero, M.P. 2009. Iron absorption from meat pate fortified with ferric pyrophosphate in iron-deficient women. Nutrition, 25 (1), 2024.

Navas-Carretero, S., Pérez-Granados, A.M., Sarri, B., Schoppen, S., Vaquero, M.P. 2007. Iron bioavailability from pate enriched with encapsulated ferric pyrophosphate or ferrous gluconate in rats. Food Science and Technology International, 13 (2), 159-163.

Noori, S., Zeynali, F., Almasi, H. 2018. Antimicrobial and antioxidant efficiency of nanoemulsionbased edible coating containing ginger (Zingiber officinale) essential oil and its effect on safety and quality attributes of chicken breast fillets. Food Control 84:312-20.

Ojha, K.S., Perussello, C.A., García, C.Á., Kerry, J.P., Pando, D., Tiwari, B.K. 2017. Ultrasonicassisted incorporation of nano-encapsulated omega-3 fatty acids to enhance the fatty acid profile of pork meat. Meat Science, 132, 99-106. 
Ouyang, Q., Liu, Y., Chen, Q., Guo, Z., Zhao, J., Li, H. and Hu, W. 2017. Rapid and specific sensing of tretacycline in food using a novel upconversion aptasensor. Food control 81, 156-163.

Ozogul, Y., Durmus, M., Ucar, Y., Ozogul, F., and Regensteın, J. M. 2016. Comparative study of nanoemulsions based on commercial oils (sunflower, canola, corn, olive, soybean, and hazelnut oils): Effect on microbial, sensory, and chemical quality of refrigerated farmed sea bass. Innovative Food Science and Emerging Technologies 33:422-30.

Ozogul, Y., Durmus, M., Uçar, Y., Koşker, A. R., and Ozogul, F. 2017a. The combined impact of nanoemulsion based on commercial oils and vacuum packing on the fatty acid profiles of sea bass fillets. Journal of Food Processing and preservation, 41 (6) e13222, 1-13

Ozogul, Y., Yuvka, I., Ucar, Y., Durmus, M., Köske, A. R., Mustafa, O., Ozogul, F., and Regensteın, J. M. 2017b. Evaluation of effects of nanoemulsion based on herb essential oils (rosemary, laurel, thyme and sage) on sensory, chemical and microbiological quality of rainbow trout (Oncorhynchus mykiss) fillets during ice storage. LWT - Food Science and Technology 75:677-84.

Patel, P. D., \& Beveridge, C. (2003). In-line sensors for food process monitoring and control. In Rapid and On-Line Instrumentation for Food Quality Assurance, (pp. 215-239).

Park, I.S., Kim, N., 2006. Development of a chemiluminescent immunosensor for chloramphenicol. Anal Chim Acta 578 (1), 19-24.

Pérez-López, B. and Merkocia, A. 2011. Nanomaterials based biosensors for food analysis applications. Trends in Food Science \& Technology 22, 625-639.

Puligundla, P., Jung, J., \& Ko, S. (2012). Carbon dioxide sensors for intelligent food packaging applications. Food Control, 25(1), 328-333

Qi, Lifeng, Zirong Xu, Xia Jiang, Caihong Hu, and Xiangfei Zou. 2004. Preparation and antibacterial activity of chitosan nanoparticles. Carbohydrate Research 339 (16):2693-2700.

Rai, M., Yadav, A., Gade, A. 2009. Silver nanoparticles as a new generation of antimicrobials. Biotechnology Advances 27:76-83. 
RajeshKumar, S., Ishaq Ahmed, V.P., Parameswaran, V., Sudhakaran, R., Sarath Babu, S. and Sahul Hameed, A.S. 2008. "Potential use of chitosan nanoparticles for oral delivery of DNA vaccine in Asian sea bass (Lates calcarifer) to protect from Vibrio (Listonella) anguillarum." Fish \& Shellfish Immunology 25 (1):47-56.

Rajeshkumar, S., Venkatesan, C., Sarathi, M., Sarathbabu, V., Thomas, J., Anver Basha, K. and Sahul Hameed, AS. 2009. "Oral delivery of DNA construct using chitosan nanoparticles to protect the shrimp from white spot syndrome virus (WSSV)." Fish \& Shellfish Immunology 26 (3):429-437.

Ravichandran, M., Hettiarachchy, N.S., Ganesh, V., Ricke, S.C., Singh, S. 2011. Enhancement of antimicrobial activities of naturally occurring phenolic compounds by nanoscale delivery against Listeria monocytogenes, Escherichia coli o157:H7 and Salmonella typhimurium in broth and chicken meat system. Journal of Food Safety, 31 (4), pp. 462-471.

Rowland, C.E., Brown, C.W., Delehanty, J.B., Medintz, I.L. 2016. Nanomaterial-based sensors for the detection of biological threat agents. Materials Today. 19 (8), oct, 464-477.

Saavedra, M., Conceição, L.E.C., Helland, S., Pousão-Ferreira, P., Dinis, M.T. 2008. Effect of lysine and tyrosine supplementation in the amino acid metabolism of Diplodus sargus larvae fed rotifers. Aquaculture, 284 (1-4), 180-184.

Sanchez-Garcia, M. D., Lopez-Rubio, A., \& Lagaron, J. M. 2010. Natural micro and nanobiocomposites with enhanced barrier properties and novel functionalities for food biopackaging applications. Trends in Food Science \& Technology, 21(11), 528-536

Sebaaly, C., Greige-Gerges, H., Stainmesse, S., Fessi, H., Charcosset, C. 2016. Effect of composition, hydrogenation of phospholipids and lyophilisation on the characteristics of eugenol-loaded liposomes prepared by the ethanol injection method. Food Bioscience, 15, 110. 
Shaalan, M., Saleh, M., El-Mahdy, M., El-Matbouli, M. 2016. Recent progress in applications of nanoparticles in fish medicine: A review. Nanomedicine: Nanotechnology, Biology, and Medicine, 12 (3), pp. 701-710.

Shadman, S., Hosseini, S. E., Langroudi, H. E., and Shabani, S. 2017. Evaluation of the effect of a sunflower oil-based nanoemulsion with Zataria multiflora Boiss essential oil on the physicochemical properties of rainbow trout (Oncorhynchus mykiss) fillets during cold storage. LWT - Food Science and Technology 79:511-517.

Shahdordizadeh, M., Taghdisi, S.M., Ansari, N., Langroodi, F.A., Abnous, K and Ramezani, M. 2017. Aptamer based biosensors for detection of Staphylococcus aureus. Sensors and Actuators B $241,619-635$

Sharma, A., Sharma, U.S. 1997. Liposomes in drug delivery: Progress and limitations. International Journal of Pharmaceutics, 154, 123-140.

Sharma, C., Dhiman, R., Rokana, N., \& Panwar, H. (2017). Nanotechnology: An untapped resource for food packaging. Frontiers in Microbiology, 8(SEP).

Sharma, V. K., Yngard, R. A., Lin, Y., \& Zboril, R. (2010). Silver nanoparticles: green synthesis approaches. In Abstracts of Papers of the American Chemical Society, vol. 239): Amer chemical soc 1155 16th st, NW, Washington, DC 20036 USA.

Shi, C.Y., Deng, N., Liang, J.J., Zhou, K.N., Fu, Q.Q., Tang, Y. 2015. A fluorescent polymer dots positive readout fluorescent quenching lateral flow sensor for ractopamine rapid detection. Analytica Chimica Acta, 854, 202-208.

Singh, H. 2016. Nanotechnology applications in functional foods; Opportunities and challenges. Preventive Nutrition and Food Science, 21, 1-8.

Singh, P.K., Jairath, G., Ahlawat.S.S. 2016. Nanotechnology: a future tool to improve quality and safety in meat industry. Journal of Food Science and Technology 53 (4), 1739-1749. 
Solval, Kevin Mis, Luis A. Espinoza Rodezno, Marvin Moncada, J. David Bankston, and Subramaniam Sathivel. 2014. Evaluation of chitosan nanoparticles as a glazing material for cryogenically frozen shrimp. LWT - Food Science and Technology 57 (1):172-180.

Smolander M., Hurme E., Koivisto M. \& Kivinen S. (2004). PCT International Patent Application. WO, 102185, A1.

Sorrentino, A., Gorrasi, G., \& Vittoria, V. (2007). Potential perspectives of bio-nanocomposites for food packaging applications. Trends in Food Science and Technology, 18(2), 84-95.

Sotelo-Boyás, M., Z. Correa-Pacheco, S. Bautista-Baños, and Y. Gómez y Gómez. 2017. Release study and inhibitory activity of thyme essential oil-loaded chitosan nanoparticles and nanocapsules against foodborne bacteria. International Journal of Biological Macromolecules 103:409-414.

Stark, B., Pabst, G., Prassl, R. 2010. Long-term stability of sterically stabilized liposomes by freezing and freeze-drying: Effects of cryoprotectants on structure. European Journal of Pharmaceutical Sciences, 41 (3-4), 546-555.

Suo, B., Li, H., Wang, Y., Li, Z., Pan, Z., \& Ai, Z. 2016. Effects of ZnO nanoparticle-coated packaging film on pork meat quality during cold storage. Journal of the Science of Food and Agriculture, 97(7), 2023-2029.

Taladrid, D., Marín, D., Alemán, A., Álvarez-Acero, I., Montero, P., Gómez-Guillén, M.C. 2017. Effect of chemical composition and sonication procedure on properties of food-grade soy lecithin liposomes with added glycerol. Food Research International, 100, 541-550.

Tan, C., Xia, S., Xue, J., Xie, J., Feng, B., Zhang, X. 2013. Liposomes as vehicles for lutein: Preparation, stability, liposomal membrane dynamics, and structure. Journal of Agricultural and Food Chemistry, 61, 8175-8184.

Toker, R.D., Kayaman-Apohan, N., Kahraman, M.V., 2013. UV curable nano-silver containing polyurethane based organic-inorganic hybrid coatings. Progress in Organic Coatings 76, $1243-1250$. 
Torres-Vargas. O.L., J. García-Salcedo, K.J. Figueroa-López. 2016. Influence of garlic nanoparticles in hamburger physicochemical characteristics. Vitae 23 (1): S512-S517.

Tran, T.T.H., Do, T.M.H., Hoang, M.H., Nguyen, D.T., Le, Q.T., Nguyen, D.N., Ngo, T.T. 2015. Rapid and sensitive detection of clenbuterol using a fluorescence nanosensor based on diazo coupling mechanism. Advances in Natural Sciences: Nanoscience and Nanotechnology, 6 (2), 1-5, June 2015, Article number 025007.

Umuhumuza, L.C. and Sun X. 2011. Rapid detection of pork meat freshness by using L-cysteinemodified gold electrode. Eur Food Res Technol. 232:425-431

[USFDA] United States Food and Drug Administration. 2014. Guidance for industry: assessing the effects of significant manufacturing process changes, including emerging technologies on the safety and regulatory status of food ingredients and food contact substances, including food ingredients that are color additives. Available from: http://www.fda.gov/Food/GuidanceRegulation/GuidanceDocumentsRegulatoryInformation / IngredientsAdditivesGRASPackaging/ ucm300661.htm> (accessed 05.11.2014).

Wang, G., Wang, T. 2008. Oxidative Stability of Egg and Soy Lecithin as Affected by Transition Metal Ions and pH in Emulsion. Journal of Agricultural and Food Chemistry, 56, 11424-11431.

Wang, Z., Liu, F., Lu, C. 2014. Evolution of biogenic amine concentrations in foods through their induced chemiluminescence inactivation of layered double hydroxide nanosheet colloids. Biosensors and Bioelectronics, 60, 237-2.

Wyser, Y., Adams, M., Avella, M., Carlander, D., Garcia, L., Pieper, G., Rennen, M., Schuermans, J., \& Weiss, J. 2016. Outlook and Challenges of Nanotechnologies for Food Packaging. Packaging Technology and Science, 29(12), 615-648.

Wu, C., Wang, L., Hu, Y., Chen, S., Liu, D., and Ye, X. 2016. Edible coating from citrus essential oilloaded nanoemulsions: Physicochemical characterization and preservation performance. The Royal Society of Chemistry 6:20892-900. 
Xiao-e, L., Green, A. N. M., Haque, S. A., Mills, A., \& Durrant, J. R. 2004. Light-driven oxygen scavenging by titania/polymer nanocomposite films. Journal of Photochemistry and Photobiology A: Chemistry, 162(2-3), 253-259.

Xiao-wei, H., Xiao-bo, Z., Ji-yong, S., Yanin, G., Jie-wen, Z., Jianchun, Z. and Lim, H. 2014.

Determination of pork spoilage by colorimetric gas sensor array based on natural pigments. Food Chemistry 145, 549-554

Xiao-Wei, H., Zhi-Hua, L., Xiao-Bo, Z., Ji-Yong, S., Han-Ping, M., Jie-Wen, Z., Li-Min, H., Holmes, M. 2016. Detection of meat-borne trimethylamine based on nanoporous colorimetric sensor arrays. Food Chemistry, 197, 930-936.

Yang, J., Ciftci, O.N. 2017. Encapsulation of fish oil into hollow solid lipid micro- and nanoparticles using carbon dioxide. Food Chemistry 231:105-113.

Yang, L., \& Li, Y. 2006. Simultaneous detection of Escherichia coli 0157:H7 and Salmonella Typhimurium using quantum dots as fluorescence labels. Analyst, 131(3), 394-401.

Yang, X., Zhou, X., Zhu, M. and Xing, D. 2017. Sensitive detection of Listeria monocytogenes based on highly efficient enrichment with vancomycin-conjugated brush-like magnetic nanoplatforms. Biosensors and Bioelectronics 91, 238-245.

Yausheva, E.V., Miroshnikov, S.A., Kosyan, D.B., Sizova, E.A. 2016. Nanoparticles in combination with amino acids change productive and immunological indicators of broiler chicken. Sel'skokhozyaistvennaya Biologiya, 51 (6), pp. 912-920.

Yazgan, H., Ozogul, Y., Durmuş, M., Balikçi, E., Gökdoğan, S., Uçar, Y., and Aksun, E.T. 2017. Effects of oil-in-water nanoemulsion based on sunflower oil on the quality of farmed sea bass and gilthead sea bream stored at chilled temperature $\left(2 \pm 2^{\circ} \mathrm{C}\right)$. Journal of Aquatic Food Product Technology 26, 8:979-992.

Yin; T., Park; J.W. 2014a. Effects of nano-scaled fish bone on the gelation properties of Alaska pollock surimi. Food Chemistry 150:463-468. 
Yin; T., Park; J.W. 2014b. Gelling properties of surimi as affected by the particle size of fish bone. LWT - Food Science and Technology 58:412-416.

Youssef, A.M., Abdel-Aziz, M.S. 2013. Preparation of polystyrene nanocomposites based on silver nanoparticles using marine bacterium for packaging. Polymer-Plastics Technology and Engineering, 52, 607-613.

Zhang, C., Yin, A. X., Jiang, R., Rong, J., Dong, L., Zhao, T., Sun, L. D., Wang, J., Chen, X., \& Yan, C. H. (2013). Time-temperature indicator for perishable products based on kinetically programmable Ag overgrowth on Au nanorods. ACS Nano, 7(5), 4561-4568.

Zhang, Z., Duan, F., He, L., Peng, D., Yan, F., Wang, M., Zong, W., Jia, C. 2016. Electrochemical clenbuterol immunosensor based on a gold electrode modified with zinc sulfide quantum dots and polyaniline. Microchimica Acta, 183 (3), 1089-1097 